Draft VERSion MARCh 5, 2022

Preprint typeset using $\mathrm{LAT}_{\mathrm{E}} \mathrm{X}$ style emulateapj v. 12/16/11

\title{
SLIPPING MAGNETIC RECONNECTION DURING AN X-CLASS SOLAR FLARE OBSERVED BY SDO/AIA
}

\author{
J. DuDÍK ${ }^{1}$
}

RS Newton International Fellow, DAMTP, CMS, University of Cambridge, Wilberforce Road, Cambridge CB3 0WA, United Kingdom

M. JANVIER

Department of Mathematics, University of Dundee, Dundee DD1 4HN, Scotland, United Kingdom

G. Aulanier

LESIA, Observatoire de Paris, UMR 8109 (CNRS), 92195 Meudon Principal Cedex, France

G. Del Zanna

DAMTP, CMS, University of Cambridge, Wilberforce Road, Cambridge CB3 0WA, United Kingdom

M. KARLICKÝ
Astronomical Institute of the Academy of Sciences of the Czech Republic, Fričova 298, 25165 Ondřejov, Czech Republic

H. E. MASON

DAMTP, CMS, University of Cambridge, Wilberforce Road, Cambridge CB3 0WA, United Kingdom

\author{
B. SCHMIEDER \\ LESIA, Observatoire de Paris, UMR 8109 (CNRS), 92195 Meudon Principal Cedex, France \\ Draft version March 5, 2022
}

\begin{abstract}
We present SDO/AIA observations of an eruptive X-class flare of July 12, 2012, and compare its evolution with the predictions of a 3D numerical simulation. We focus on the dynamics of flare loops that are seen to undergo slipping reconnection during the flare. In the AIA $131 \AA$ observations, lower parts of $10 \mathrm{MK}$ flare loops exhibit an apparent motion with velocities of several tens of $\mathrm{km} \mathrm{s}^{-1}$ along the developing flare ribbons. In the early stages of the flare, flare ribbons consist of compact, localized bright transition-region emission from the footpoints of the flare loops. A DEM analysis shows that the flare loops have temperatures up to the formation of Fe XXIV. A series of very long, S-shaped loops erupt, leading to a CME observed by STEREO. The observed dynamics are compared with the evolution of magnetic structures in the "standard solar flare model in 3D". This model matches the observations well, reproducing both the apparently slipping flare loops, S-shaped erupting loops, and the evolution of flare ribbons. All of these processes are explained via 3D reconnection mechanisms resulting from the expansion of a torus-unstable flux rope. The AIA observations and the numerical model are complemented by radio observations showing a noise storm in the metric range. Dm-drifting pulsation structures occurring during the eruption indicate plasmoid ejection and enhancement of reconnection rate. The bursty nature of radio emission shows that the slipping reconnection is still intermittent, although it is observed to persist for more than an hour.
\end{abstract}

Keywords: Sun: flares - Sun: X-Rays, gamma rays - Sun: UV radiation - Sun: Radio radiation magnetic reconnection - Magnetohydrodynamics (MHD)

\section{INTRODUCTION}

Solar flares are the most energetic manifestation of solar magnetic activity. They are characterized by a rapid increase in emission over a broad range of the electromagnetic spectrum, from X-rays and extreme-ultraviolet (EUV) to radio wavelengths (e.g., Kane 1974; Fletcher et al. 2011, White et al. 2011). A typical Hare encom-

J.Dudik@damtp.cam.ac.uk mjanvier@maths.dundee.ac.uk

${ }^{1}$ DAPEM, Faculty of Mathematics Physics and Computer Science, Comenius University, Mlynská Dolina F2, 84248 Bratislava, Slovakia passes a wealth of dynamical phenomena that are manifestations of the release of magnetic energy via the process of magnetic reconnection (Parker 1957; Sweet 1958, Priest \& Forbes 2000; Zweibel \& Yamada 2009). One of the most distinct signatures of these dynamical phenomena is that of the formation of flare loops, which emit strongly in X-rays and EUV (e.g., Fletcher et al. 2011), and the accompanied flare ribbons prominent in EUV and up to visible wavelengths (e.g., Warren \& Warshall 2001). Eruptive flares also exhibit large-scale restructuring of the magnetic field accompanied by coronal mass ejections (CMEs) that possibly result from the expul- 
sion of coronal magnetic flux ropes (e.g., van Ballegooijen \& Martens 1989; Amari et al. 2000; Moore et al.|2001; Green \& Kliem 2009: Green et al.|2011; Patsourakos et al. 2013) and are an important driver of space weather.

Based on the observations of eruptive flares, various models have been developed to describe and interpret their main features. The standard 2D CSHKP model (Carmichael 1964, Sturrock 1966, Hirayama 1974, Kopp \& Pneuman 1976), for example, describes the formation of Hare loops and the flux rope (e.g., Dere et al. 1999; Cheng et al. 2013), from the reconnection of coronal magnetic field lines. Magnetic reconnection releases magnetic energy in the form of particle acceleration and thermal energy. Energetic particles guided along the magnetic field can impact the chromosphere (e.g., Reid et al.|2012), where they lead to the formation of flare ribbons (e.g., Schmieder et al. 1996). The chromosphere can also be heated by thermal conduction (Petkaki et al.|2012). Both processes lead to chromospheric evaporation (Neupert 1968), which quickly fills the flare loops (e.g., Raftery et al. 2009; Milligan \& Dennis 2009; Brosius \& Holman 2010 Del Zanna et al. 2011b; Ning 2011; Doschek et al. 2013 Brosius 2013). The high density in the flare loops makes them a prominent emission structures. Flare ribbons are the footpoints of these loops, and their emission comes from compact transition region features (e.g., Graham et al. 2011; Young et al. 2013) and the underlying chromosphere.

While the CSHKP model provides a straightforward picture of eruptive flares, it fails at describing the intrinsically 3D features of solar flares. Examples of such features include e.g. motion of EUV or X-ray sources parallel to the flare ribbons (e.g. Inglis \& Gilbert 2013), or the evolution of the twist or shear of coronal loops, which is important for the dynamics of both flare loops and the flux rope (Aulanier et al. 2012). Other models, for example the tether cutting model (Moore et al. 1997; Fan 2012 ) or torus-unstable flux rope models (Torök et al. 2004 Aulanier et al. 2010) have been proposed to extend the standard model for eruptive flares to 3D. In particular, the 3D numerical simulation of a torus-unstable flux rope (Aulanier et al. 2012, Janvier et al. 2013) has helped to shed light on the underlying reconnection mechanism forming both flare loops and the flux rope. The absence of 3D null-points means that the reconnection takes place in Quasi-Separatrix Layers (QSLs), and in particular in the thinning high current density region underneath the expanding flux rope.

The QSLs (Priest \& Démoulin 1995, Démoulin et al. 1996, 1997) are locations where the mapping of the magnetic field lines exhibits strong gradients, but is not discontinuous. This means that there are no separatrices dividing the magnetic field into regions of different connectivities, as is the case with magnetic null-points. Rather, connectivity domains are bordered by regions where the connectivity is strongly changing, but is still continuous. The magnetic field distortion is analytically measured by calculating the norm $N$ of the field line mapping (Démoulin et al. 1996) or the squashing degree $Q$ (Titov et al. 2002; Pariat \& Démoulin 2012). Because of the distortion of the magnetic field, high electric current density regions can form where the distortion is the strongest (e.g., Aulanier et al. 2005; Masson et al. 2009 Wilmot-Smith et al.2009). 'This therefore results in mag- netic reconnection as ideal MHD can break down (Parker 1957: Sweet 1958: Priest \& Forbes 2000).

In the case of QSLs, magnetic field lines passing through them can undergo successive reconnection that is seen as an apparent "flipping", or "slipping" motion (Priest \& Démoulin 1995 Priest et al. 2003 Aulanier et al. 2006). The apparent slipping motion is a consequence of the local diffusion in the reconnection region, where the neighbouring field lines continually exchange connectivities (Aulanier et al. 2006). Because of the local rotation of the magnetic field vector within the coronal diffusion region, this process induces apparent field line velocities all along their length that can be different from the plasma velocity (Priest et al. 2003, Aulanier et al. 2006). Slipping reconnection is theoretically predicted in both eruptive and confined flares, as investigated in Masson et al. (2012). Janvier et al. (2013) showed that the speed of the apparent motion of the field lines can be directly linked to the norm $N$ and the reconnection rate. This study provided an important insight on reconnection mechanisms in 3D and the link with magnetic topology. Since the QSLs are more general than true null-points which require discontinuities, slipping reconnection is a more general mechanism of energy release not only during solar flares, but also in active regions (Aulanier et al. 2007).

At first, current sheets required for reconnection can be formed dynamically, both in 2D (e.g., Magara et al. 1996 Lin \& Forbes 2000, Bárta et al. 2011) and also in 3D (e.g., Antiochos et al. 1999; Lynch et al. 2008; Aulanier et al. 2010; Kliem et al. 2010, Aurass et al. 2011). Then, the current layer itself can be destabilized, leading to the formation of several plasmoids and null-points moving along the sheet (e.g., Loureiro et al. 2012). Merging and fragmentation of plasmoids then results in smaller current sheet systems that can be associated with fast reconnection regimes, providing the necessary energy release rate and acceleration of particles (Shibata \& Tanuma 2001; Karlický 2004; Bárta et al. 2008; Uzdensky et al. 2010; Bárta et al. 2011; Karlický \& Bárta 2011; Karlický et al. 2012). These processes can be observed in X-rays as the formation, ejection and interaction of plasmoids Ohyama \& Shibata 1998; Kołomański \& Karlický 2007, Milligan et al. 2010) and in the dm-radio range as drifting pulsation structures (DPS, Kliem et al. 2000, Karlický et al. 2002; Karlický 2004; Karlický \& Bárta 2007; Karlický et al. 2010). Magnetic reconnection at high coronal altitudes can also manifest itself as a radio noise storm (Elgaroy 1977; Del Zanna et al. 2011a).

Based on the above, the general expectation is that during eruptive flares, slipping motion of the field lines should manifest itself as an apparent slipping motion of flare loops, and plasmoid emission in the form of DPS should be observed at radio wavelengths as a consequence of both the flux rope eruption and the reconnection regime involving dynamics of the current layer. To the author's knowledge, these processes have not previously both been reported in the same flare. Moreover, reports of the slipping reconnection itself are rare Aulanier et al. 2007; Testa et al. 2013) and none are associated with flares.

In this paper, we report on the X1.4 flare of July 12, 2012 that manifests both the slipping reconnection and in later phase the fast reconnection with plasmoids (DPSs). 
AIA observations of the flare are described in Sect. 2 This section contains an overview of the events during the flare together with analysis of the apparent slipping motion of the flare loops. Individual contributions to flare EUV emission as observed by AIA are obtained from DEM analysis in Sect. 3. Section 4 presents the results of an earlier numerical simulation reproducing the expansion of an unstable asymmetric flux rope. With the simulated region having similar features as the observed active region 11520 , the simulation is directly compared to observations. Evolution of the QSLs and the flux rope are also described along with the slipping reconnection process. In Sect. 5, we report on radio emission associated with the flare. A Summary and Conclusions are given in Sect. 6.

\section{SDO/AIA OBSERVATIONS AND DATA ANALYSIS}

An X1.4 flare occurred on 2012 July 12 in the large complex of active regions NOAA 11519, 11520 and 11521. It was an eruptive, long-duration event with a peak in the GOES 1-8A flux at 16:49 UT (Fig. 1), but with flaring activity starting as early as 15:00 UT. The bulk of the flare EUV and X-ray emission (Sect. 2.1) occurred in AR 11520 (Hale class $\beta \gamma \delta / \beta \gamma \delta$ ), with one ribbon extending to AR $11521(\beta \gamma / \beta)$. The magnetogram for these active regions is shown in Fig. 1. The small, old AR 11519 $(\alpha / \alpha)$ located further $100^{\prime \prime}$ westward was not involved in the flare. Altogether, the active region complex spanned nearly $40^{\circ}$ in solar longitude.

\subsection{Overview}

The Atmospheric Imaging Assembly (AIA, Lemen et al. 2012, Boerner et al. 2012) on board the Solar Dynamics Observatory (SDO) consists of 4 identical, normal-incidence two-channel telescopes providing multiple, near-simultaneous full-Sun images with both high temporal resolution and high spatial resolution $\left(1.5^{\prime \prime}\right.$, pixel size $\left.0.6^{\prime \prime}\right)$. AIA images of the Sun are taken in 10 filters, 7 of which are centered on EUV wave-

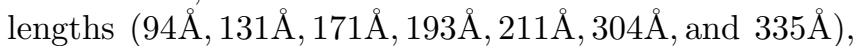
and 3 on UV or visible wavelengths $(1600 \AA, 1700 \AA$, and $4500 \AA)$. The EUV filters are centered on some of the strongest lines in the solar EUV spectrum. Such multifilter AIA observations allow a study of the thermal structure of the solar atmosphere. However, the presence of a variety of emission lines originating at different temperatures within one filter bandpass makes the temperature responses of the AIA EUV filters (Fig. 2) highly multithermal in nature (e.g., O'Dwyer et al. 2010, Del Zanna et al. 2011c; Schmelz et al. 2013). 'This behaviour is also reflected in the responses of the $131 \AA$ and $94 \AA$ filters used for flare observations. Their responses are double-peaked due to sensitivity to both flare and coronal temperatures. The response of the AIA $131 \AA$ filter peaks at $\log (T / K)=5.75$ and again at 7.05 due to contributions from Fe VIII and Fe XXI, respectively. The main contributors to the response of the $94 \AA$ filter are Fe $\mathrm{X}$ and Fe XVIII, producing peaks at $\log (T / K)=6.0$ and 6.85 , respectively. Fe XIV contributes to this filter as well (Del Zanna 2013). Nevertheless, the flare emission observed by AlA is now well understood (Petkaki et al. 2012 Del Zanna 2013). Together with its high temporal and spatial resolution, AIA is well-suited for studies of
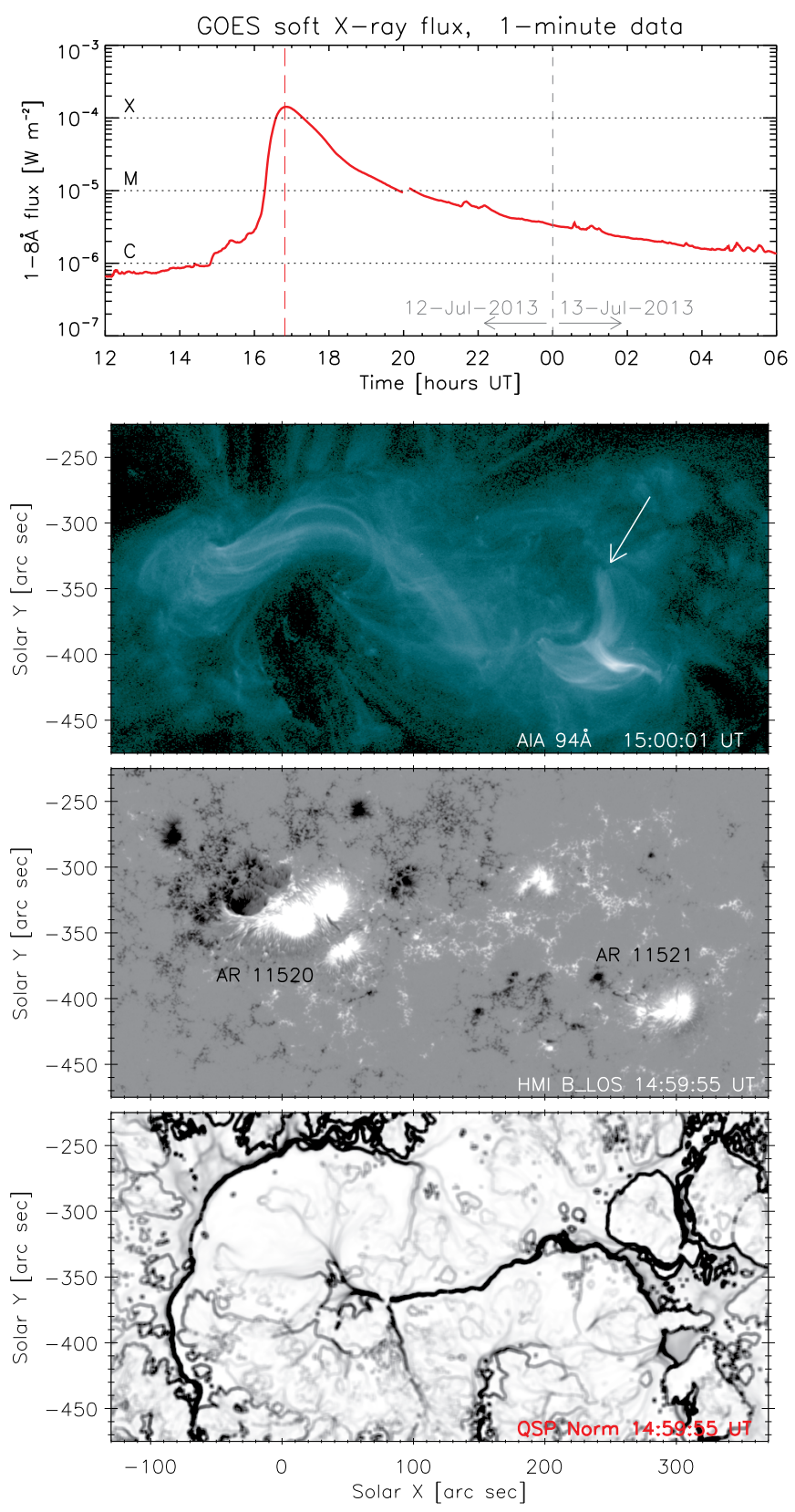

Figure 1. Top: Evolution of the X-ray flux in the $1-8 \AA$ channel observed by GOES. Second row: Pre-flare coronal configuration in the SDO/AIA $94 \AA$ filter showing a large sigmoid in AR 11520 and a brightening in AR 11521. Footpoints of one of the loop systems participating in the brightening are denoted by a white arrow. Third row: Portion of the longitudinal SDO/HMI magnetogram showing the active region complex. The AR 11519 is not shown as it lies further to the West. Bottom: Intersections of the large-scale quasi-separatrix layers with the photosphere, calculated from a potential extrapolation of the magnetic field.

dynamical phenomena that occur on small scales, such as the evolution of individual features during a flare.

The flare evolution, as observed by SDO/AIA, is summarized in Fig. 3 and in Table 1 . which should act as a reference guide for the online movies, as well as individual events during the flare discussed in this paper.

2.1.1. Pre-flare state, brightening in AR 11521 and the large-scale magnetic topology 
Table 1

Summary of individual events during the X1.4 flare. Time and locations are approximate, as the events are dynamical.

\begin{tabular}{|c|c|c|c|}
\hline Approx. Time [UT] & Event description & Location [Solar $X, Y]$ & Notes \\
\hline $14: 48-15: 15$ UT & Brightening of loop systems in AR 11521 & {$\left[+300^{\prime \prime},-400^{\prime \prime}\right]$} & Fig. 1 second row \\
\hline 15:00 UT & Flare start in AR 11520 (first flare loop) & {$\left[0^{\prime \prime},-300^{\prime \prime}\right]$, above $\mathrm{F} 1$} & Arrow 1 in Fig. 3 top left \\
\hline 15:00 UT & Radio noise storm beginning at $200-500 \mathrm{MHz}$ & & Fig. 18 type III-like bursts \\
\hline $15: 01-15: 34$ UT & Flare loops undergo slipping motion and gradual brightening & {$\left[-20^{\prime \prime},-320^{\prime \prime}\right]$} & Sect. 2.2.1 Figs. 67 \\
\hline 15:07-16:10 UT & Growing system of hot loops in $131 \AA$ (erupts later) & {$\left[+100^{\prime \prime},-330^{\prime \prime}\right]$} & Arrow 2 in Fig. 3 (rows $2-4$ ) \\
\hline $15: 43-16: 07 \mathrm{UT}$ & Second slipping event, gradual developing of NR & {$\left[-20^{\prime \prime},-320^{\prime \prime}\right]$} & Sect. 2.2 .2 Figs. 89 \\
\hline 16:02-16:17 UT & Hot $131 \AA$ loop appearing at the end of NRH & {$\left[-100^{\prime \prime},-320^{\prime \prime}\right]$} & Arrow 3 in Fig. 3 fourth row \\
\hline 16:05-16:25 UT & Brightening and development of the $\mathrm{NRH}$ & {$\left[-100^{\prime \prime},-320^{\prime \prime}\right], \mathrm{NRH}$} & Fig. 3 fifth row \\
\hline 16:05-16:35 UT & Third slipping event along developing NRH & {$\left[-50^{\prime \prime},-320^{\prime \prime}\right]$} & Sect.2.2.3 Figs. 1011 \\
\hline $16: 13-16: 33 \mathrm{UT}$ & Large intensity enhancement along NR moving westward & {$\left[-30^{\prime \prime},-270^{\prime \prime}\right], \quad \mathrm{NR}$} & Fig. 3 万fth row \\
\hline $16: 14-16: 27 \mathrm{UT}$ & PRH brightening, slipping assoc. with eruption in $131 \AA$ & {$\left[+150^{\prime \prime},-360^{\prime \prime}\right], \mathrm{PRH}$} & Fig. 3 fifth row, Figs. 1213 \\
\hline $16: 16 \mathrm{UT}$ & Start of a strong radio flare & & Sect. 2.2 Figs. $1818 \square \square$ \\
\hline 16:30-17:00 UT & PRH widens, with associated coronal dimming & {$\left[+200^{\prime \prime},-350^{\prime \prime}\right]$} & Fig. 3 sixth row \\
\hline $16: 34-16: 40 \mathrm{UT}$ & Deformation of the $\mathrm{NRH}$ & {$\left[-90^{\prime \prime},-320^{\prime \prime}\right]$} & \\
\hline $16: 40-17: 00 \mathrm{UT}$ & $\mathrm{NRH}$ extension in N-S direction, assoc. coronal dimming & {$\left[-100^{\prime \prime},-350^{\prime \prime}\right]$} & Fig. 3 sixth row \\
\hline 16:49 UT & Peak of the GOES $1-8 \AA$ X-ray flux & & Fig. 1 top \\
\hline
\end{tabular}

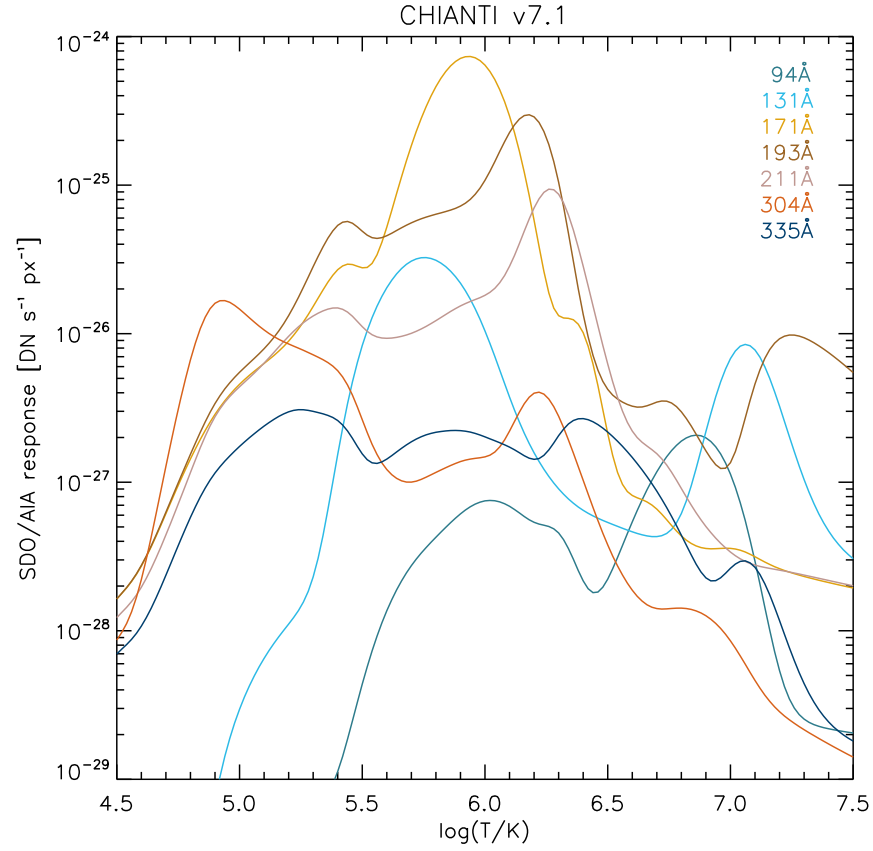

Figure 2. Temperature responses of the AIA EUV filters, calculated using $\log \left(n_{\mathrm{e}} / \mathrm{cm}^{3}\right)=9$ and the abundances from Schmelz et al. (2012).

The magnetic configuration of the AR 11520 before the flare is that of a forward S-shaped sigmoid visible only in AIA $94 \AA$ (Fig. 1, second row). This sigmoid overlies an active region filament $\mathrm{F} 1$ visible in AIA $304 \AA$ (Fig. 3 . top right).

In the neighbouring AR 11521, a brightening of several loop systems occurs at around 14:48, i.e., shortly before the flare in AR 11520. One of the loop systems involved in the brightening is rooted in the vicinity of the position $[X, Y]=\left[+250^{\prime \prime},-330^{\prime \prime}\right]$ (arrow in Fig. 1). This position corresponds to the leftmost extension of the positive-polarity ribbon $(\mathrm{PR})$ during the flare, and also to an intersection of one of the strongest QSLs with the photosphere (Fig. 1. bottom).

We obtained these QSL footpoints from a potential extrapolation of a SDO/HMI (Helioseismic Magnetic Imager, Scherrer et al. 2012) magnetogram using the method of Alissandrakis (1981) and Gary (1989). The potential extrapolation is an approximative method which assumes that there are no electric currents in the region. Clearly, this assumption is not valid either during the flare or near the vicinity of a sigmoid. Nevertheless, it can be used to infer the number and approximate shape of the large-scale QSLs that separate the magnetic flux closed within the active region complex from other closed, or locally "open" magnetic field lines (as also done in e.g., Chandra et al. 2009). We found that there are two strong, large-scale QSLs in the active region complex (Fig. 1. bottom): one semi-circular shaped on the lefthand side of the image in the negative polarities, and a second one at $Y \approx-350^{\prime \prime}$ in the positive polarities. A portion of the QSL in the negative polarities is shifted to the north with respect to the footpoint locations of the sigmoidal loops. Such mismatch can be expected because of the electric currents present in the sigmoid.

As already noted, the positive-polarity QSL at $Y \approx$ $-350^{\prime \prime}$ corresponds well to the ribbon PR involved the flare (Fig. 3). This QSL provides the spatial connection between the flare in AR 11520 and the brightening in AR 11521 occuring immediately before the flare. We note that flaring, erupting or even radio events closely related in time have higher than a random occurrence (Wheatland 2006), which could be a result of a "domino effect" (Chifor et al. 2006). Events related in time can have spatial connections through the magnetic field and its topology (e.g. Zuccarello et al. 2009, Liu et al. 2009. Jiang et al. 2011; Töök et al. 2011; Shen et al. 2012, Mészárosová et al. 2013: Schrijver et al. 2013). Therefore, the brightening in AR 11521 is the earliest signature of the flaring activity in the entire AR complex.

\subsubsection{Flare evolution}

The flare itself starts at approximately 15:00 UT, when the first flare loop can be clearly identified in the AIA $131 \AA$ filter (Arrow 1 in Fig. 3). This is the first signature of high temperature flare emission in any of the AIA filters. At one end, the loop is rooted near $\left[+40^{\prime \prime},-350^{\prime \prime}\right]$ in the QSL in the strongest positive-polarity spots in AR 11520. It then encircles the western spot counterclockwise from SW to NE and is rooted on the other end in the negative polarity. The loop is highly sheared and lies along the outer edge of the curved filament F1 (Fig. 3. top right). The $131 \AA$ flare loop is easily identifiable with one of the pre-existing sigmoidal loops.

Over the next 40 minutes, the flare gradually devel- 

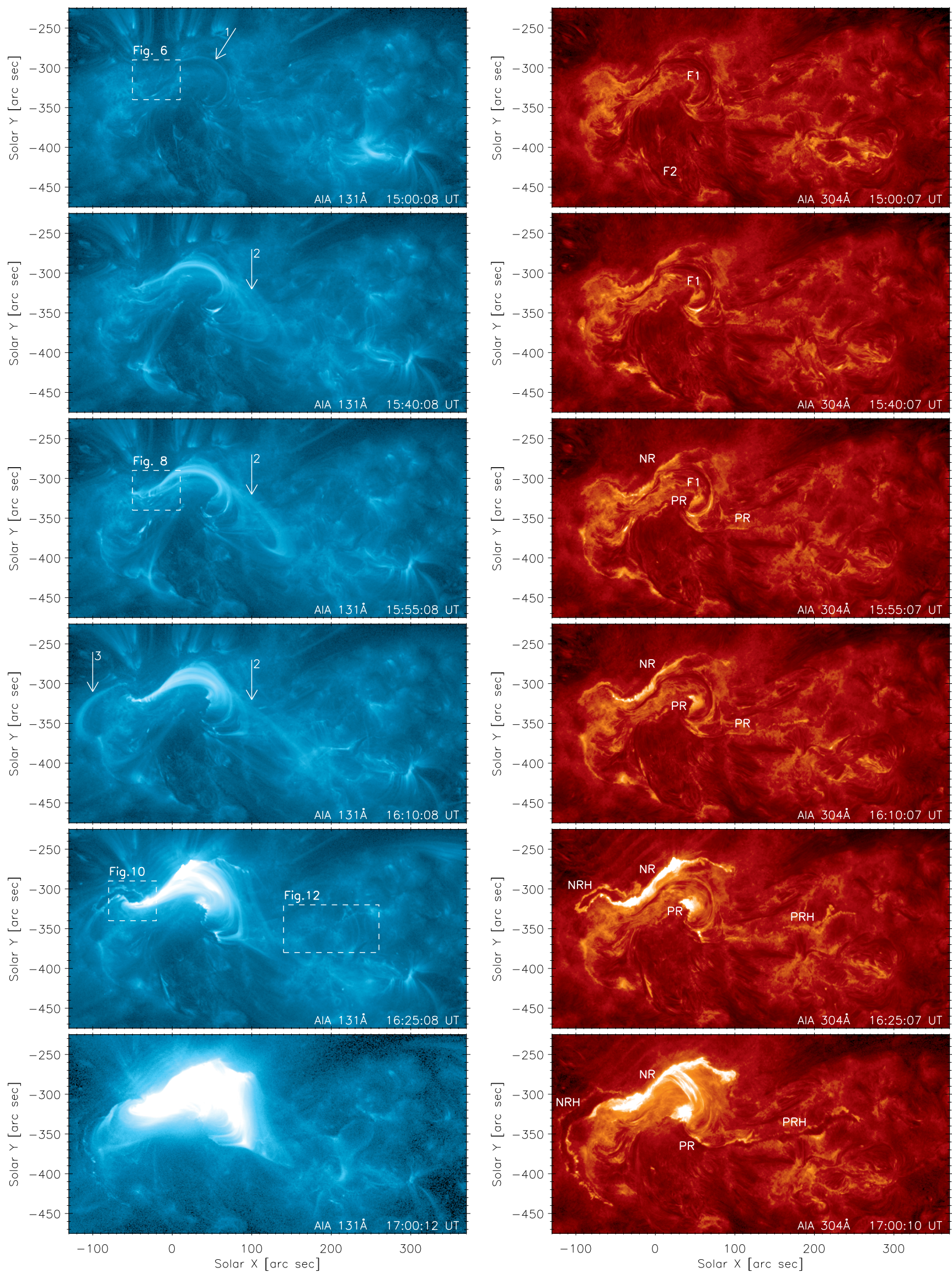

Figure 3. Overview of the evolution of the X1.4 flare on July 12, 2012. Arrow 1 denotes the first flare loop visible. Arrow 2 points to the erupting hot loops, while Arrow 3 denotes the loop appearing at the end of the NRH. Individual features involved in the flare are marked. See the text for details. Boxes enclose areas for more detailed time-series shown in the figures indicated. The intensities are scaled logarithmically. The images are available as mpeg animations (Movies 1 and 2) in the online version. 

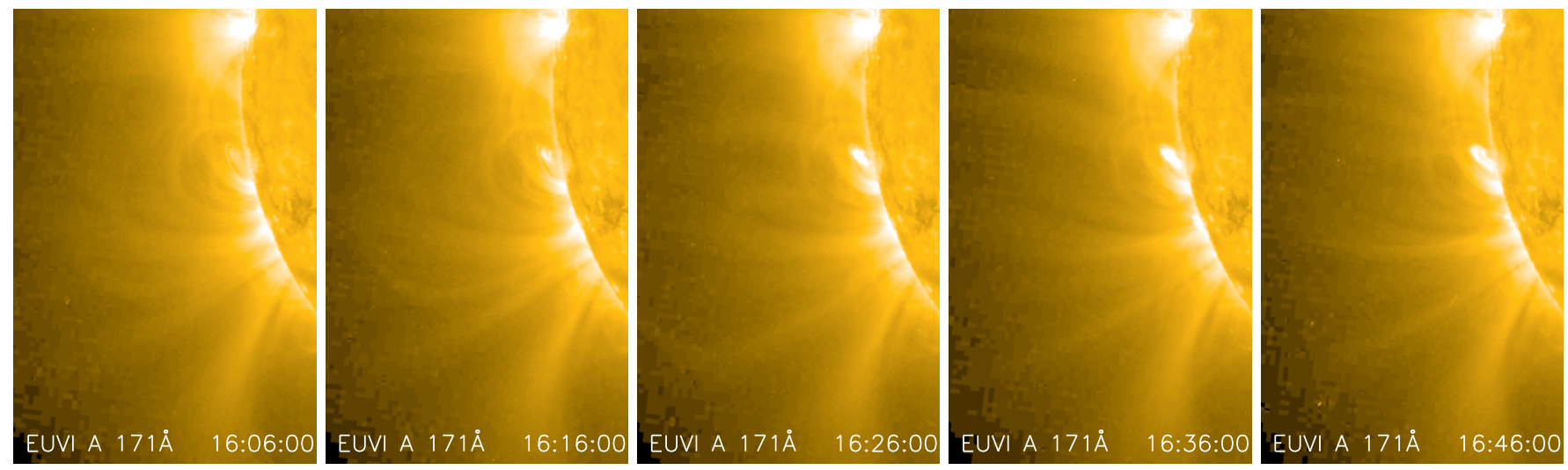

Figure 4. CME observed by the STEREO-A SECCHI/EUVI telescope in the 171Å channel. The separation angle between STEREO-A spacecraft and Earth was $+120.0^{\circ}$.
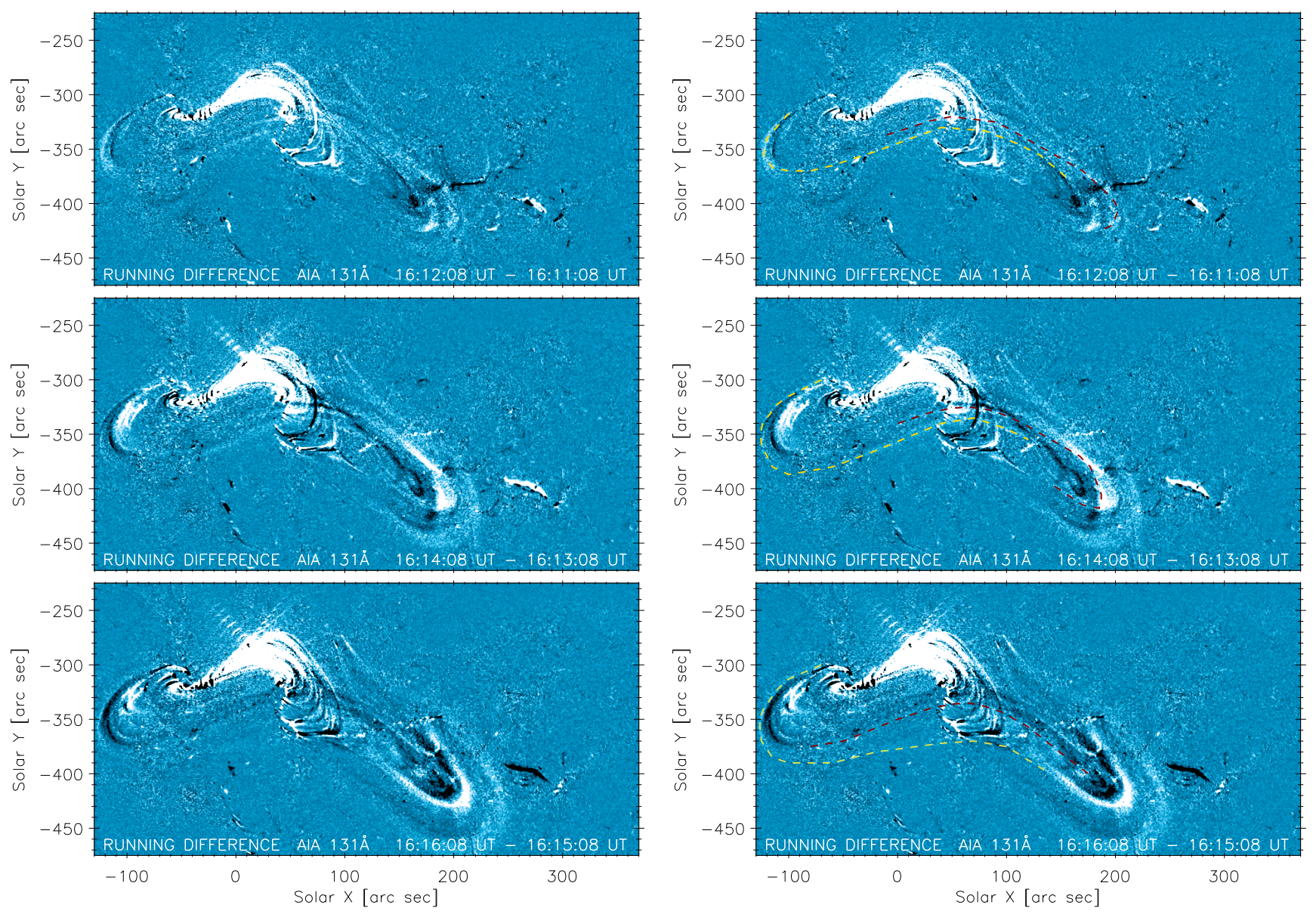

Figure 5. AIA $131 \AA$ images running-difference images with time delay of 1 min, saturated to $\pm 20 \mathrm{DN} \mathrm{s}^{-1} \mathrm{px}^{-1}$. Portions of two long erupting loops are outlined by colored lines in the right images.

ops into a rather compact, highly sheared bundle of flare loops. Some of the flare loops undergo expansion in the SW direction (Arrow 2 in Fig. 3). Their footpoints gradually move along the QSL which develops into the positive-polarity ribbon (PR) and its hook (PRH, Fig. 3 second to fifth row). The apparent motion of the footpoints along the PR accelerates, and by around 16:25, the loops have erupted. This eruption is observed by the twin STEREO satellites as a CME (Fig. 4) consisting of expanding concentric coronal loops in $171 \AA$. Unfortunately, the STEREO/EUVI instrument (Wuelser et al.
$2004)$ does not contain "hot" flare filters, so the erupting loops cannot be directly identified in STEREO observations. The expansion of the $171 \AA$ loops can be driven by the erupting hot loops, as reported for another event by Zhang et al. (2012).

The arcade of flare loops meanwhile continues to widen, with the flare ribbons brightening and growing in lateral directions (Fig. 3, second to fifth row). These extensions and brightenings are often in the form of bright blobs in $304 \AA$ or $1600 \AA$ moving along the ribbons, associated with the apparent slippage of flare loops. This is 


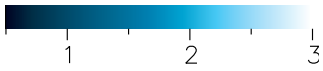
$\log _{10}\left(D N s^{-1} p x^{-1}\right)$
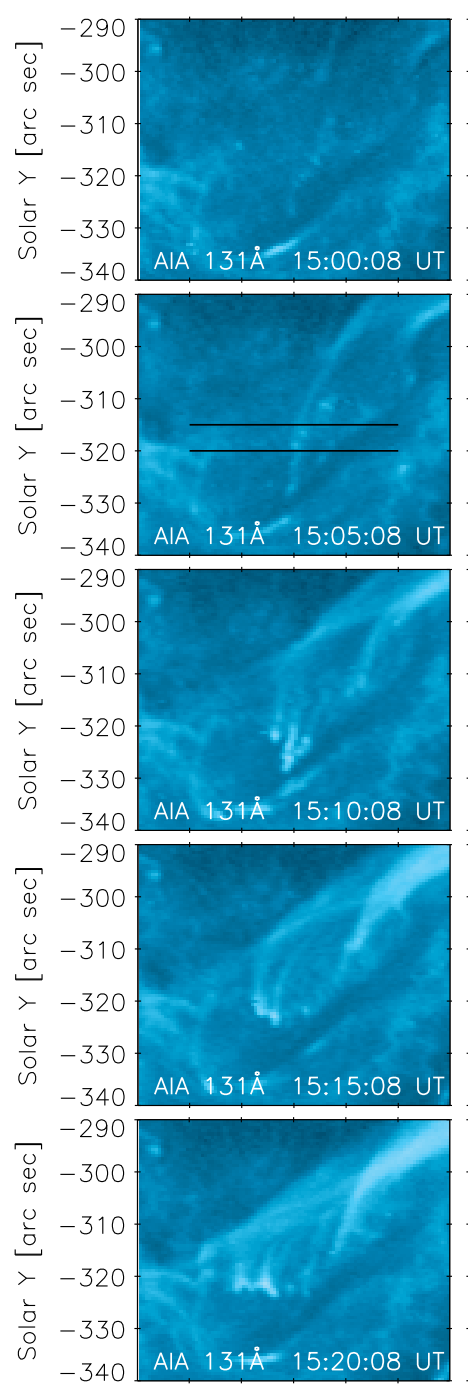

-40-30-20-10 0 Solar X [arc sec]
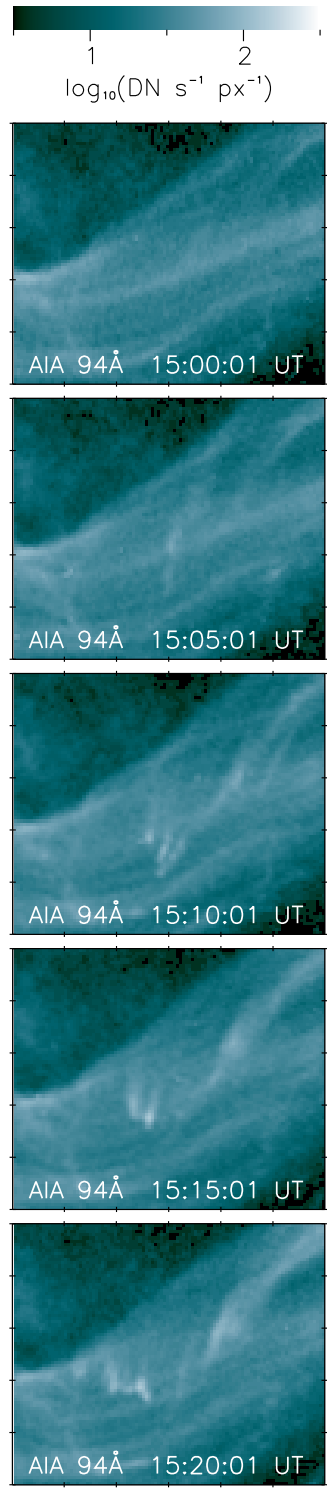

$-40-30-20-100$

Solar X [arc sec]
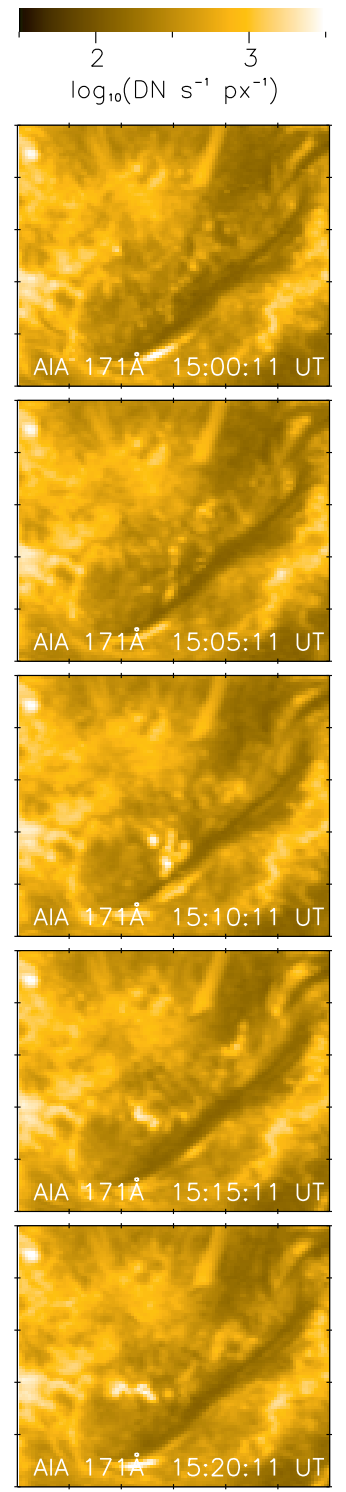

$-40-30-20-100$

Solar $X[\operatorname{arc~sec}]$

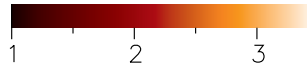

$\log _{10}\left(D N s^{-1} p x^{-1}\right)$
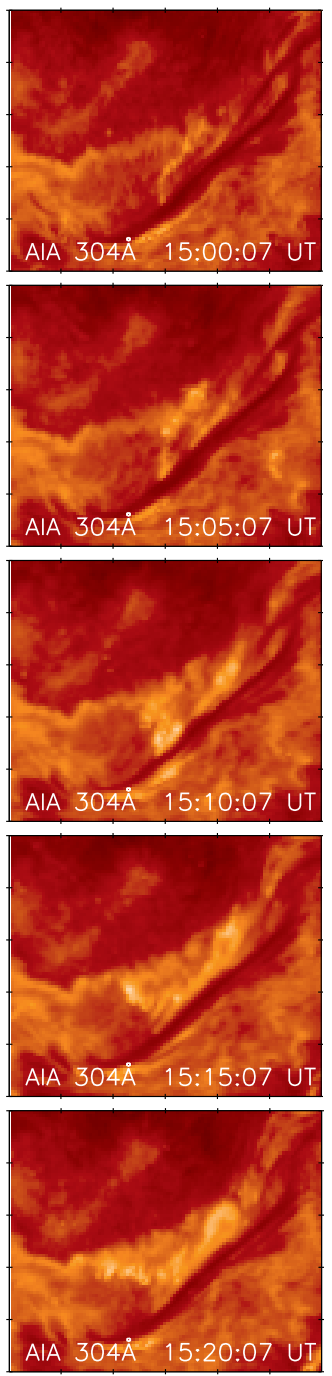

-40-30-20-10 0

Solar X [arc sec]

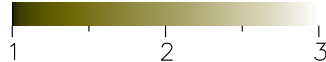

$\log _{10}\left(D N s^{-1} p x^{-1}\right)$
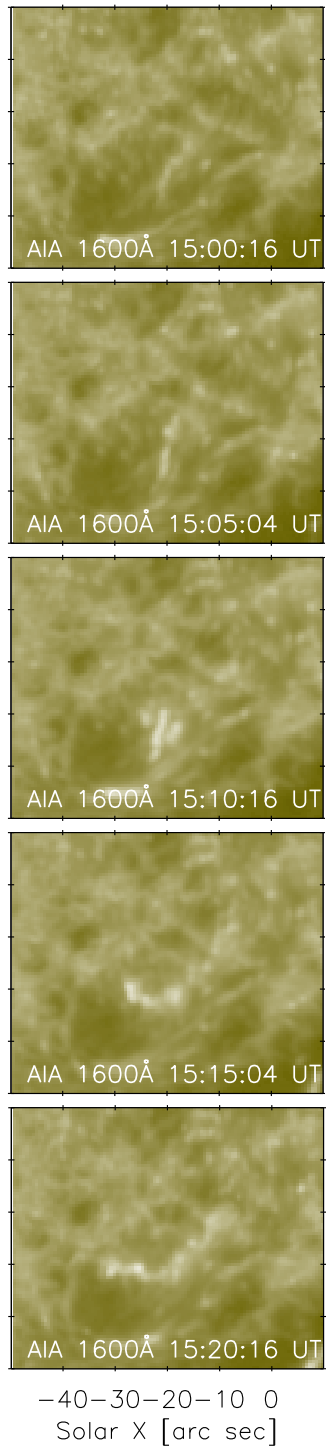

Figure 6. Slipping magnetic loops at the beginning of the flare. Dark lines in the top left panel show positions of the cuts used to construct $X$-t plots (stackplots) shown in Fig. 7 The intensities are scaled logarithmically, with units of $\mathrm{DN} \mathrm{s}^{-1} \mathrm{px}^{-1}$. An animation of the AIA $131 \AA$ observations (left column) is available as the online Movie 4.

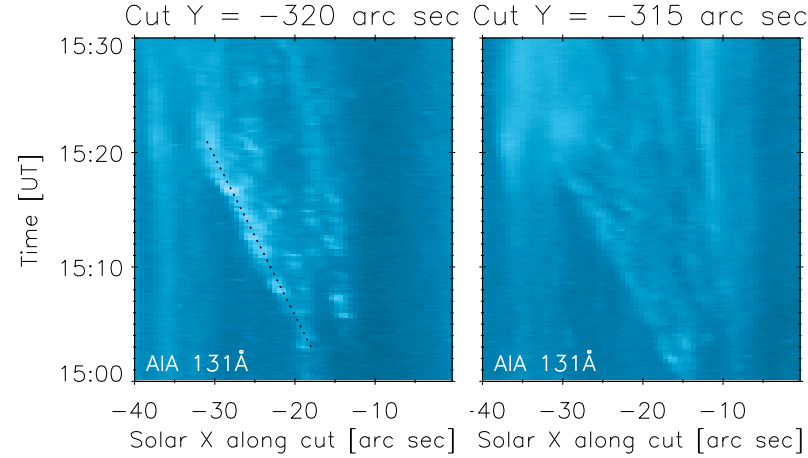

Figure 7. Stackplots along the two cuts plotted in Fig. 6 showing slipping loops in the AIA 131 Åfilter. The black line on the left image corresponds to the velocity of $8.7 \mathrm{~km} \mathrm{~s}^{-1}$.

discussed in detail in Sect. 2.2. Around 16:25 UT, the ribbons are the most prominent emitting structures in the $304 \AA$ observations (dominated by He II). Both ribbons exhibit hooks on their ends. These hooks are typically not as bright as the rest of the ribbon (Fig. 3. fifth row). The hooks show a quite rapid evolution with the eruption. For example, the hook of the negative-polarity ribbon (NRH) undergoes a deformation starting around 16:34 UT and subsequently extends by more than $100^{\prime \prime}$ in the N-S direction grazing along the large-scale QSL (shown in Fig. 1. bottom). The width of both the NRH and PRH increases over time to a maximum of about 20 $30^{\prime \prime}$. Therefore, even if their width is increasing, they still stay rather narrow. Both of them also stay continuous and enclose narrow regions of coronal dimming observed in all AIA EUV filters.

This coronal dimming confirms that the erupting loops are rooted in both hooks. In fact, a bright loop arc lo- 


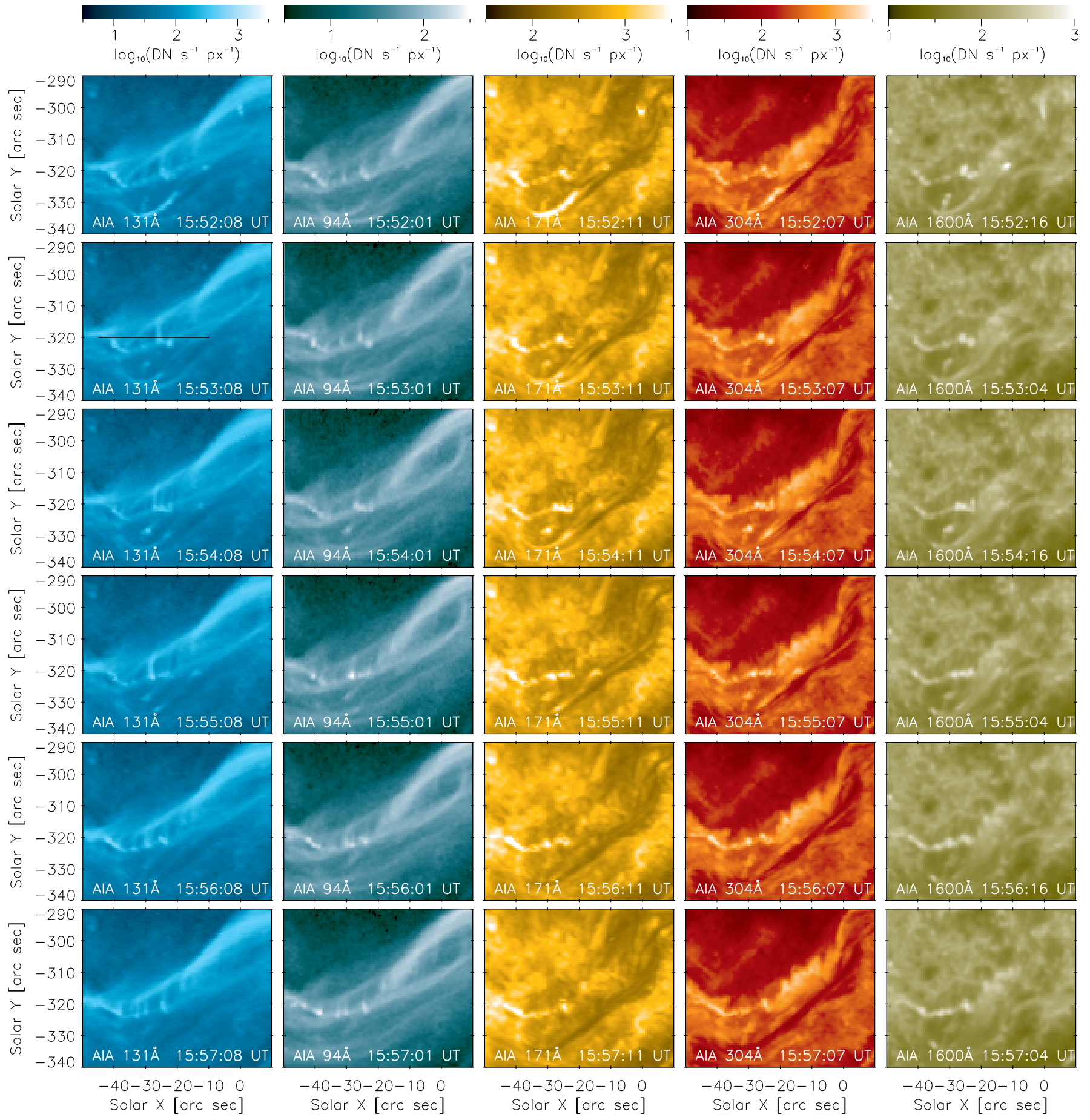

Figure 8. Slipping magnetic loops during the second slipping event. Dark lines in the top left panel show positions of the cuts used to construct $X$-t plots (stackplots) shown in Fig. 9 The intensities are scaled logarithmically, with units of $\mathrm{DN} \mathrm{s}^{-1} \mathrm{px}^{-1}$. An animation of the AIA $131 \AA$ observations (left column) is available as the online Movie 5.

cated at the end of NRH can be identified around 16:0216:17 UT (Arrow 3 in Fig. 33). This bright loop arc is a portion of a system of long loops with projected length of more than 250" (Fig. 5). These long loops are a part of the erupting structure. Because these loops are very long, for most of their length they are barely visible in the AIA $131 \AA$ observations due to low signal caused in turn by decreasing density along the loops. However, the motion of this loop system can be discerned in Fig. 5 or in the corresponding online Movie 3, where the running- difference of the $131 \AA$ observations with time lag of $1 \mathrm{~min}$ is shown. To guide the eye, the discernible portions of two long loops are outlined in Fig. 55, right by yellow and dark red lines. The running-difference also shows a series of other moving loops following the outlined ones. We emphasize that these long loops have general S-shaped appearance, and so are presumably non-potential. Unfortunately, due to the overlay of many emitting structures, the footpoints of these loops in the PRH cannot be traced with confidence. 


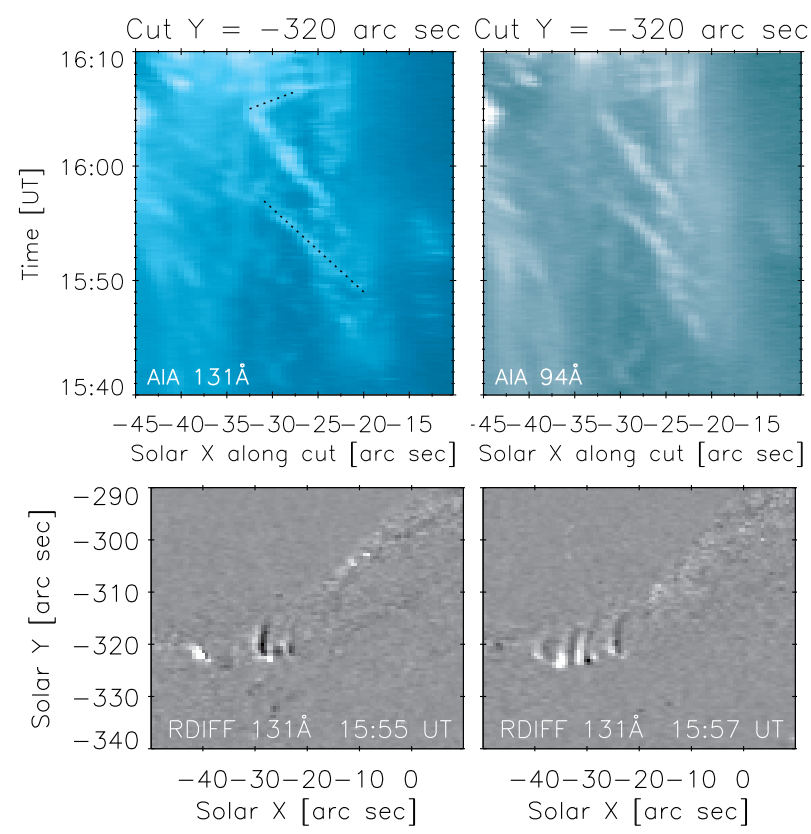

Figure 9. Top: $X$ - $t$ stackplots along the cut plotted in Fig. 8 showing apparently slipping loops in the AIA $131 \AA$ and $94 \AA$ filters. The long, dotted black line on the top left image corresponds to the velocity of $16.6 \mathrm{~km} \mathrm{~s}^{-1}$, while the short dotted line corresponds to velocity of $4.5 \mathrm{~km} \mathrm{~s}^{-1}$. Bottom: Examples of running-difference images at 15:55:32 and 15:57:32 UT showing one (bottom left) and multiple (bottom right) slipping loops.

One of the interesting features of this flare is that the filament F1, as well as other filaments within the AR complex (e.g. F2, Fig. 3, top right) do not erupt during the flare. Most importantly, F1 is still present and visible at 16:10-16:25 UT, i.e., the time of eruption of the hot loops. This suggests that F1 is constituted by a portion of the magnetic field not participating in the eruption and/or the flare. We note that the intensity of F1 as observed in the $304 \AA$ filter is highly variable with time, although there are little or no morphological changes. This is due to the fact that the He II emission originates from scattering (e.g., Andretta et al. 2003 Labrosse et al. 2010, and references therein). The scattering increases with the ribbon emission. An additional contribution comes from the diffraction pattern showing many secondary maxima (Poduval et al. 2013).

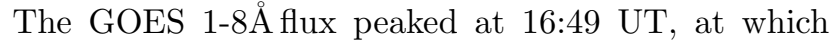
time the $\mathrm{CME}$ is in the interplanetary space and the arcade of flare loops is well developed and is cooling (Fig. 3 bottom).

\subsection{Individual slipping events}

We now focus on the apparent slipping motion of the flare loops observed in $131 \AA$. This motion is observed throughout the early stage of the flare. Four times, when the apparent slipping motion is most prominent, can be identified. They are listed in Table 1 and discussed in the next subsections. Note that the plasma velocity can be decoupled from the velocity of the apparent motion of the field lines (Priest et al. 2003). Hereafter, we use the terms "apparent slipping motion" or "slipping motion" to describe the apparent motion of the flare loops as observed by AIA. The physical origin of the apparent slipping motion of flare loops is further discussed in Sect.
4.3 .

\subsubsection{5:00-15:34 UT}

The first signature of apparent slipping motion of the flare loops is observed immediately at the start of the flare. The slipping is best visible in the negative-polarity footpoints of the first flare loops. Part of the timesequence of this event is shown in Fig. 6 and in the online Movie 4. This figure is a zoom in the region NR indicated in Fig. 3. It shows AIA observations in filters $131 \AA, 94 \AA$, $171 \AA, 304 \AA$ and $1600 \AA$, with a time step of $5 \mathrm{~min}$. The apparent slipping motions are best seen in $131 \AA$ and are weak in $94 \AA$. All other filters show only concentrated enhancements of emission consisting of several point-like features. This means that this emission originates in the rather compact transition region near the loop photospheric footpoints (similarly as in Graham et al. 2011. Young et al. 2013). In other words, the loops emit primarily in Fe XXI and not in Fe VIII or Fe IX, meaning that their temperature is around $10 \mathrm{MK}$. We examine this point in detail in Sect. 3. We emphasize here that the transition region emission seen in all AIA filters is the first signature of the developing ribbon NR. During these early stages of the flare, the ribbon emission is only due to the footpoints of these dense, hot flare loops.

We note that some of the footpoints are very close to F1, especially around 15:05-15:10 UT. The F1 does not exhibit any significant structural changes, suggesting that its magnetic field is stable even to perturbations of the surrounding field as close as $1-2^{\prime \prime}$. Similarly, the sigmoid in $94 \AA$ remains largely unperturbed during this time (Fig. 6. Column 2), except for the widening of the arcade and apparent slippage of the flare loops.

To study the apparent slipping motion of the hot flare loops, we construct stackplots along artificial "cuts" inserted at $Y=-315$ and $-320^{\prime \prime}(X-t$ plots). These cuts are shown in Fig. 6 as dark lines. The stackplots are shown in Fig. 7. Several intensity structures can be discerned, moving in the negative $X$ direction, in agreement with a visual inspection of the time-sequence in Fig. 6. One of the brightest structures is moving with an apparent velocity of $8.7 \pm 0.3 \mathrm{~km} \mathrm{~s}^{-1}$ (dotted line in Fig. 7). The error in velocity is estimated as the error of the line slope. There are other structures exhibiting apparent motion in the same direction, but they show large and intermittent intensity variations. There is no distinguishable velocity component in the perpendicular $(Y)$ direction.

The apparent slipping motion is also evident in the online Movies 1, 2 and 4, which have full temporal cadence $(12 \mathrm{~s})$. We note that the slipping motion can easily be missed in the visual inspection of AIA movies with lower cadence (e.g., $1 \mathrm{~min}$ ).

\subsubsection{5:43-16:07 UT}

After the first time interval discussed in the previous section, the apparent slipping motion of the flare loops becomes less evident or nearly invisible. Then, a series of apparently slipping loops reappear shortly after 15:43 UT. These loops appear brighter and more dynamical than during the time interval described in Sect. 2.2.1. Figure 8 shows a portion of the time-sequence with a cadence of 1 minute. The slipping is again predominantly 


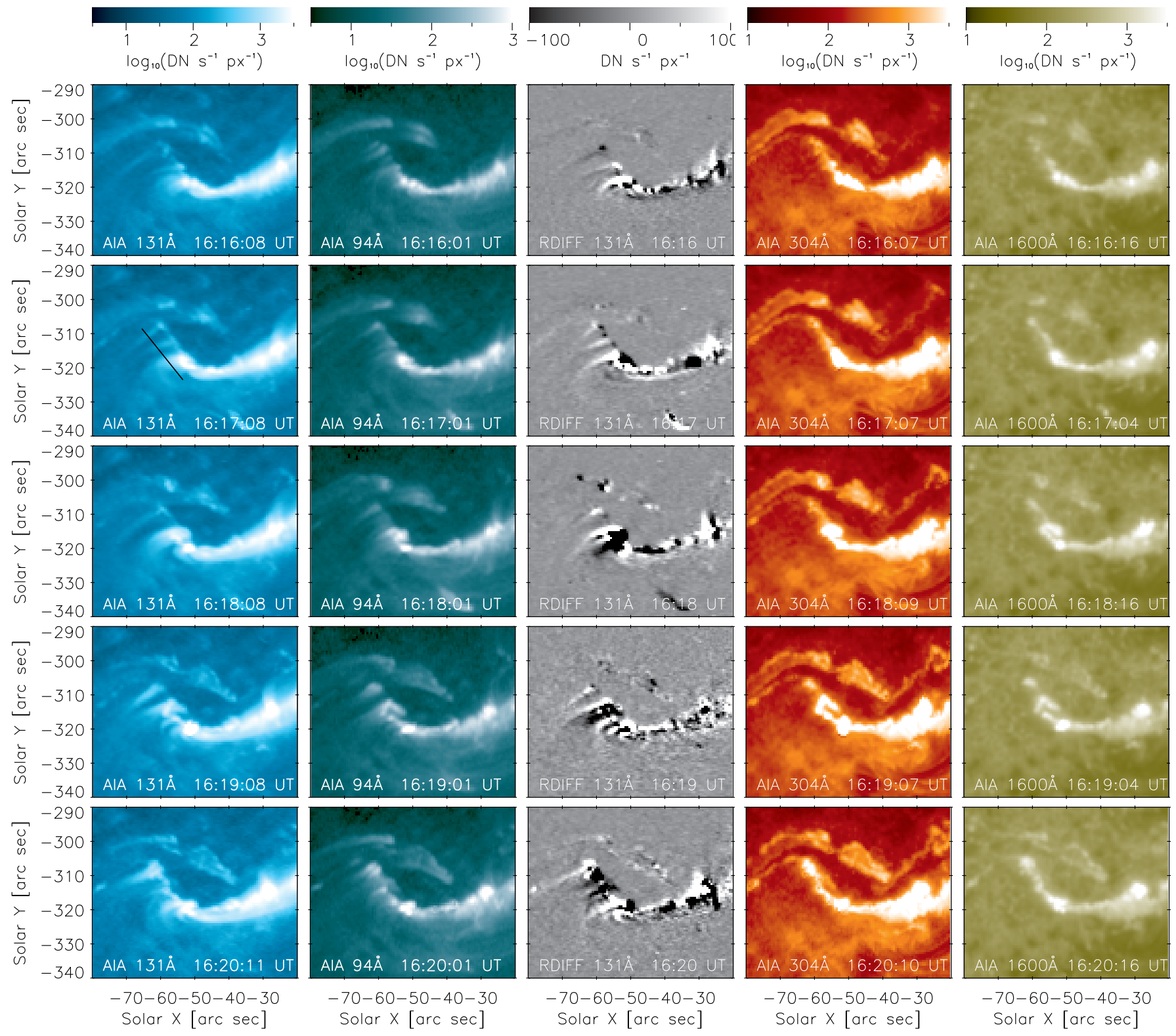

Figure 10. Example of slipping magnetic loops along the developing NR/NRH. Dark lines in the top left panel show positions of the cuts used to construct $S$ - $t$ plots (stackplots) shown in Fig. 11 The intensities are scaled logarithmically, except the AIA $131 \AA$ running difference in the middle column. An animation of the AIA $131 \mathrm{~A}$ observations (left column) is available as the online Movie 6.

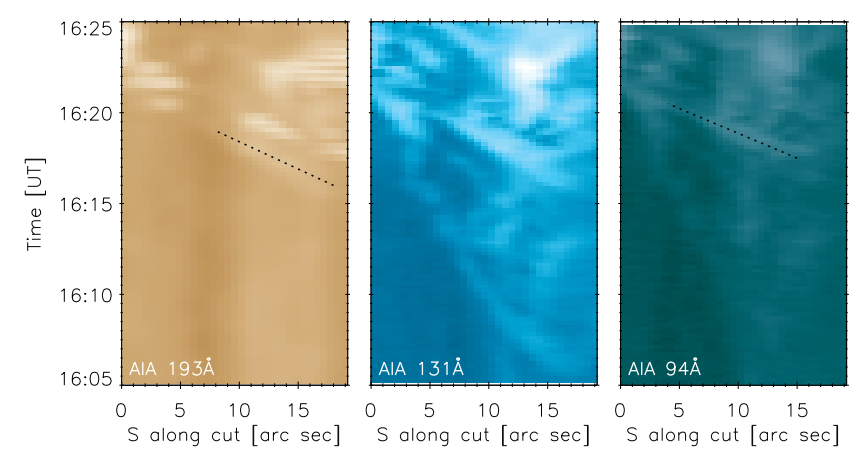

Figure 11. $S$ - $t$ stackplots along the cut plotted in Fig. 10 showing slipping loops in the AIA $193 \AA 131 \AA$ and $94 \AA$ filters. The dotted black line on the $193 \AA$ stackplot (left) corresponds to the velocity of $40 \mathrm{~km} \mathrm{~s}^{-1}$, while the dotted line on the $94 \AA$ stackplot (right) stands for $44 \mathrm{~km} \mathrm{~s}^{-1}$. in the negative $X$ direction. The intensity variations of the apparently slipping loops make it difficult to distinguish individual structures moving in the opposite direction. We again construct stackplots along an artificial cut placed at $Y=-320^{\prime \prime}$. The stackplots (Fig. 9) show a series of moving intensity features, with the one denoted by a long, dotted line having a velocity of $16.6 \pm 2 \mathrm{~km} \mathrm{~s}^{-1}$. The apparently moving loops are also clearly visible on the running-difference images (Fig. 9, bottom row) with a time delay of $12 \mathrm{~s}$. At 15:52 UT, only one intense, apparently moving loop is visible, while at 15:57 UT, there are several, as shown by the stackplots. Note also that there are indications of somewhat weaker structures moving short distances in the opposite direction. The brightest one is outlined by the short dotted short line (Fig. 9), which corresponds to a velocity of $4.5 \mathrm{~km} \mathrm{~s}^{-1}$.

At $94 \AA$, only the most intense of the $131 \AA$ loops can 

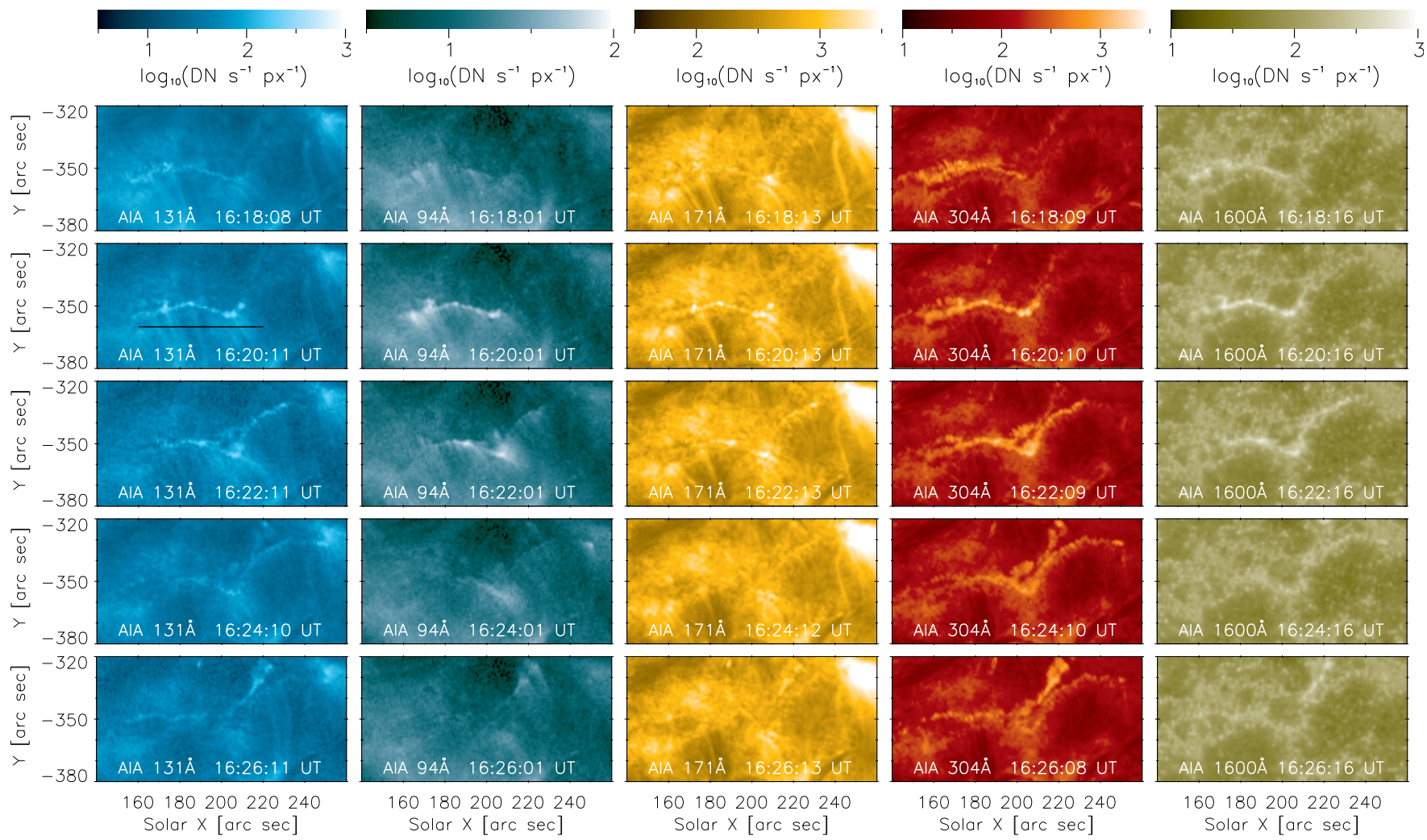

160180200220240

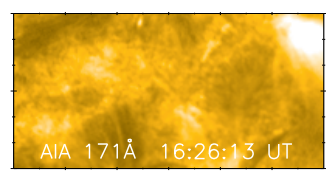

160180200220240 Solar X [arc sec]

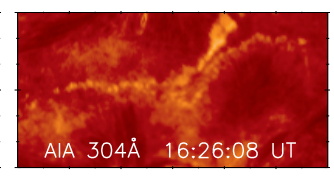

160180200220240 Solar $X$ [arc sec]

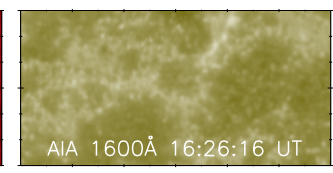

160180200220240 Solar $X[$ arc sec]

Figure 12. Fast evolution of the PRH during eruption. Dark line in the AIA 131Å image at 16:20 UT shows position of the cut used to construct $X$-t plot shown in Fig. 13 The intensities are scaled logarithmically, with units of $\mathrm{DN} \mathrm{s}^{-1} \mathrm{px}^{-1}$.

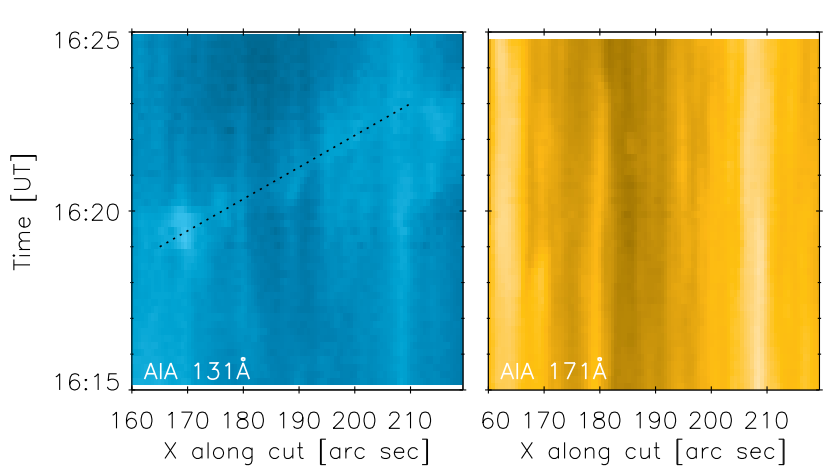

Figure 13. $X-t$ stackplot along the cut plotted in Fig. 12 showing moving structure observed in the AIA $131 \AA$, but not in AIA $171 \AA$. The dotted black line corresponds to the velocity of $136 \mathrm{~km} \mathrm{~s}^{-1}$.

be clearly distinguished (Fig. 8). These can also be seen in the $94 \AA$ stackplot shown in Fig. 9 right. There are no discernible temporal shifts between the $131 \AA$ and $94 \AA \AA$ bands, suggesting no temporally resolved cooling of the hot plasma.

The transition region emission near the footpoints of the apparently slipping loops is again visible in all AIA filters, with a clear one-to-one correspondence. The emission morphology is that of a chain of bright dots, resembling a pearl necklace in the AIA $304 \AA$ and $1600 \AA$ images at 15:57 UT. This transition region emission constitutes the elongating, developing NR.

\subsubsection{6:05-16:35 UT}

As the NR continues to develop, flare loops apparently move along it and along its hook (NRH). The most prominent example is shown in Fig. 10, with stackplots along the cut shown in Fig. 11. We denote the coordinate along the cut as $S$, measured from left to right. The stackplots show multiple structures moving in both directions, but predominantly in the negative- $S$ direction. This is not surprising, since this is the local direction of the ribbon extension. The brightest apparently moving loops are seen at $131 \AA$ and $94 \AA$. However, one of the loops is seen at $193 \AA$ and $131 \AA$ rather than at $94 \AA$, apparently moving with a velocity of $44 \pm 5 \mathrm{~km} \mathrm{~s}^{-1}$ (Fig. 11 . dotted line in the left panel). Considering the temperature responses (Fig. 2), this means that the loop emits in Fe XXIV. This occurence of a flare loop at $193 \AA$ is the only example we were able to find in this dataset. This is due to the fact that the $193 \AA$ channel is normally dominated by transition region and coronal emission from moss or warm coronal loops, with the secondary peak at $\log (T / \mathrm{K})=7.2$ which is more than an order of magnitude lower than the primary one.

\subsubsection{6:14-16:27 UT}

The bulk of the brightest flare loops are rooted in a small portion of the PR oriented in the N-S direction, directly in the strongest positive-polarity sunspots. Since the magnetic field is strong here, the footpoints of the flare loops are concentrated and any apparent slippage here is not easily distinguished.

There is however one prominent event, exhibiting moving structures along the PR and its developing hook. This event is the eruption of a portion of the hot $131 \AA$ loops, associated with a travelling brightening along the PRH (Fig. 12). This brightening is seen in 
all AIA filters simultaneously. We note especially that this brightening happens immediately after the eruption of the long loop (Fig. 5) described in Sect. 2.1. We therefore interpret it as the apparently moving footpoints of the erupting loops.

We again construct a stackplot along the artificial cut inserted at $Y=-360^{\prime \prime}$ (black line in Fig. 12). The stackplot (Fig. 13, left) shows a single weak structure moving in the positive- $X$ direction with the velocity of $136 \pm 15 \mathrm{~km} \mathrm{~s}^{-1}$ (dotted line). The vertical stripes of enhanced intensity are stationary, warm coronal loops. They are seen in the $171 \AA$, both in Fig. 12 and on the $171 \AA$ stackplot (Fig. 13 right). Where the moving $131 \AA$ feature temporarily overlies these warm coronal loops, the intensity becomes enhanced, since the emission is optically thin.

The brightening of PRH due to the apparently moving footpoints of erupting loops causes intensity variations of the warm coronal loops anchored in the PRH. As can be seen from Figs. 12 and 13 , these loops fade or disappear after the eruption.

\section{DEM ANALYSIS}

We now investigate the temperature structure of the individual structures within the flare, in particular the slipping and erupting loops. To do this, we performed a differential emission measure (DEM) analysis on each pixel of a selected AIA frame, within the field of view given by the $X=\left[-60^{\prime \prime}, 190^{\prime \prime}\right]$ and $Y=\left[-410^{\prime \prime},-270^{\prime \prime}\right]$. We selected the observations at 15:55 UT as representative of the flare (c.f. Fig. 3, third row). At this time, both the slipping loops are visible (Sect. 2.2.2, together with the erupting loops moving along the PRH appearing as a single wide bundle (Arrow 2 in Fig. 3 third row, also Fig. 14 top left). We remind the reader that the presence of plasma at flare temperatures can already be discerned by visual inspection of the AIA images, in particular the $131 \AA$ and $171 \AA$ channels (Sect. 2.2.1). The aim of the DEM reconstruction is to estimate the contribution of the Fe XVIII 93.93A, Fe XXI $128.75 \AA$ and Fe XXIV $192.02 \AA$ lines to the AIA flare bands $94 \AA, 131 \AA$ and $193 \AA$, respectively. The contribution function of these lines peaks for $\log \left(T_{\max } / \mathrm{K}\right)=6.85$, 7.05 and 7.25, respectively. Therefore, these lines sample well the contribution of the hot plasma to the AIA observations.

We note that in general, the DEM inversion problem is ill-posed and under-constrained (e.g., Craig \& Brown 1976, 1986: Judge et al. 1997). Any solution found is not unique, as it typically contains additional constraints, such as some form of regularisation or smoothing of the solution, or an a-priori assumption on the functional form of the solution. (e.g., del Zanna 1999, Aschwanden \& Boerner 2011; Hannah \& Kontar 2012). The regularized inversion of Hannah \& Kontar (2012) has been specifically adapted for DEM reconstruction of the AIA data and used e.g. to recover DEMs at each AIA pixel in an observation of an eruptive off-limb event (Hannah \& Kontar 2013). We adopt this method for DEM analysis of the selected AIA images at 15:55 UT and use the implementation provided in the dn2dem_map_pos.pro IDL routine. In the reconstruction, the photospheric abundances of Asplund et al. (2009) are used similarly as in the flare modeling of Petkaki et al. (2012). Newest atomic data benchmarked against best available solar and laboratory spectra (see Del Zanna 2013, Sect. 3 therein for details) are also used together with the atomic data from the CHIANTI database, v7.1 (Landi et al. 2013 Dere et al. 1997). We verified that the assumption of abundances and atomic data has only a small effect on the shape of the reconstructed DEMs. This is because the AIA responses are dominated by Fe ions (O'Dwyer et al. 2010: Del Zanna 2013) and the atomic data for flare lines are reliable (Petkaki et al. 2012).

Once the DEM is recovered for each AIA pixel, predicted intensity maps for each AIA filter are calculated as follows. First, the DEM obtained for a given pixel is used to calculate a corresponding synthetic spectrum. To do this, CHIANTI v7.1 is used together with the newest atomic data available. The obtained synthetic spectrum is then multiplied by the spectral response of a given AIA filter, and finally integrated in the wavelength direction. Predicted contribution of a specific spectral line to a given AIA band is calculated in a similar manner. This is done for each pixel to obtain the predicted intensity map.

We determined that the AIA observations are best reproduced using 19 temperature bins of $\log (T / K)=5.5$ 7.3. This temperature interval is chosen to adequately cover the range of temperatures observed by the AIA instrument (Fig. 2) and the many contributions to its bandpasses (O'Dwyer et al. 2010; Del Zanna 2013). The DEM reconstruction obtained for each AIA pixel results in good agreement between observed and predicted intensities for the $131 \AA, 171 \AA, 193 \AA$, and $211 \AA$ filters. An example is shown in Fig. 14, top left and top center for the AIA $131 \AA$. The $94 \AA$ and $335 \AA$ contain some areas, especially within the flare loops arcade, where the reconstructed intensities do not approximate the observed ones. At these locations, the $\operatorname{DEM}(T)$ has large horizontal errors in the $\log (T / \mathrm{K})=6.6-6.8$ temperature bins, affecting the Fe XVI and Fe XVIII contributions to the $335 \AA$ and $94 \AA$ bands, respectively. As an example, the predicted intensity map for Fe XVIII is shown in Fig.14 middle row, right. The locations of poorly recovered DEMs correspond to dark spots and patches of darker areas, especially within the flare loops arcade. Note that these locations of poorly recovered DEMs at $\log (T / K)=6.6-6.8$ correspond to locations where the LOS pierces the filament F1 and overlying flare loop arcade. The F1 shows dark as well as bright threads (e.g., Figs. 3 and 8, see also Alexander et al. 2013), while the overlying flare loops are visible in all of the AIA flare filters, namely $94 \AA, 131 \AA$ and $193 \AA$ (Fig. 14 ). We therefore suspect that the true $\operatorname{DEM}(T)$ structure in such locations is complicated, with more than two peaks, and cannot be adequately recovered by the (Hannah \& Kontar 2012 ) method due to the enforced smoothness. Including more temperature bins at lower or higher $\log (T / K)$ improves the reconstruction only marginally. Nevertheless, outside of these areas, the DEM is recovered successfully, and the results confirm strong contribution of Fe XVIII to the AIA $94 \AA$ channel.

The results of the DEM reconstruction also confirm that the observed apparently slipping flare loops are indeed emitting strongly in Fe XXI (Fig. 14, bottom), 

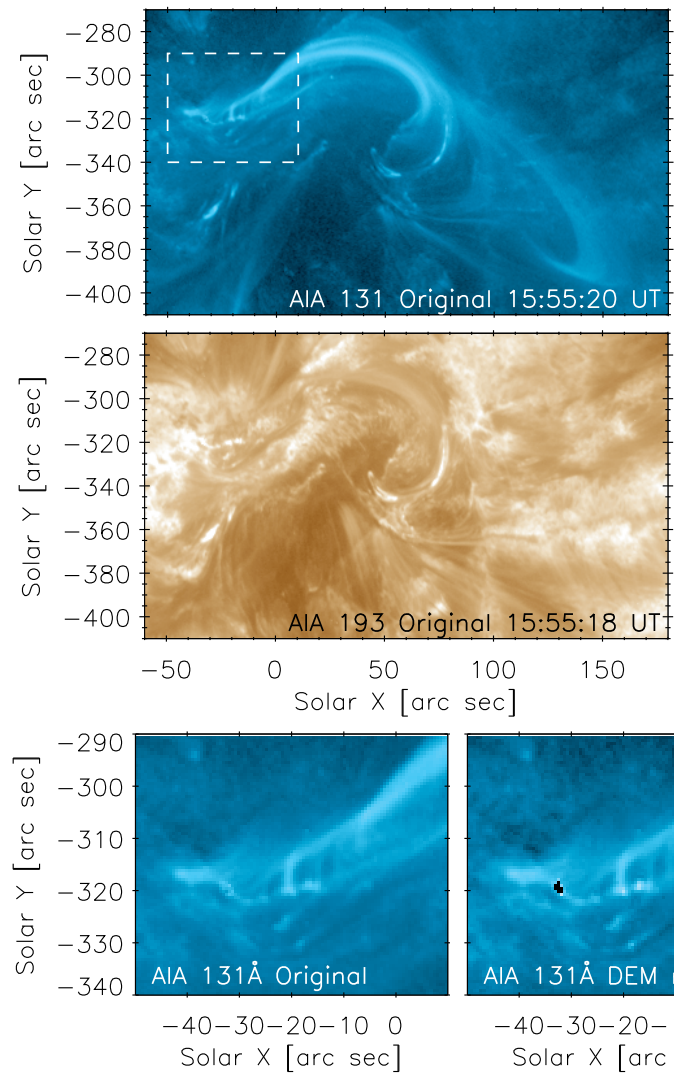
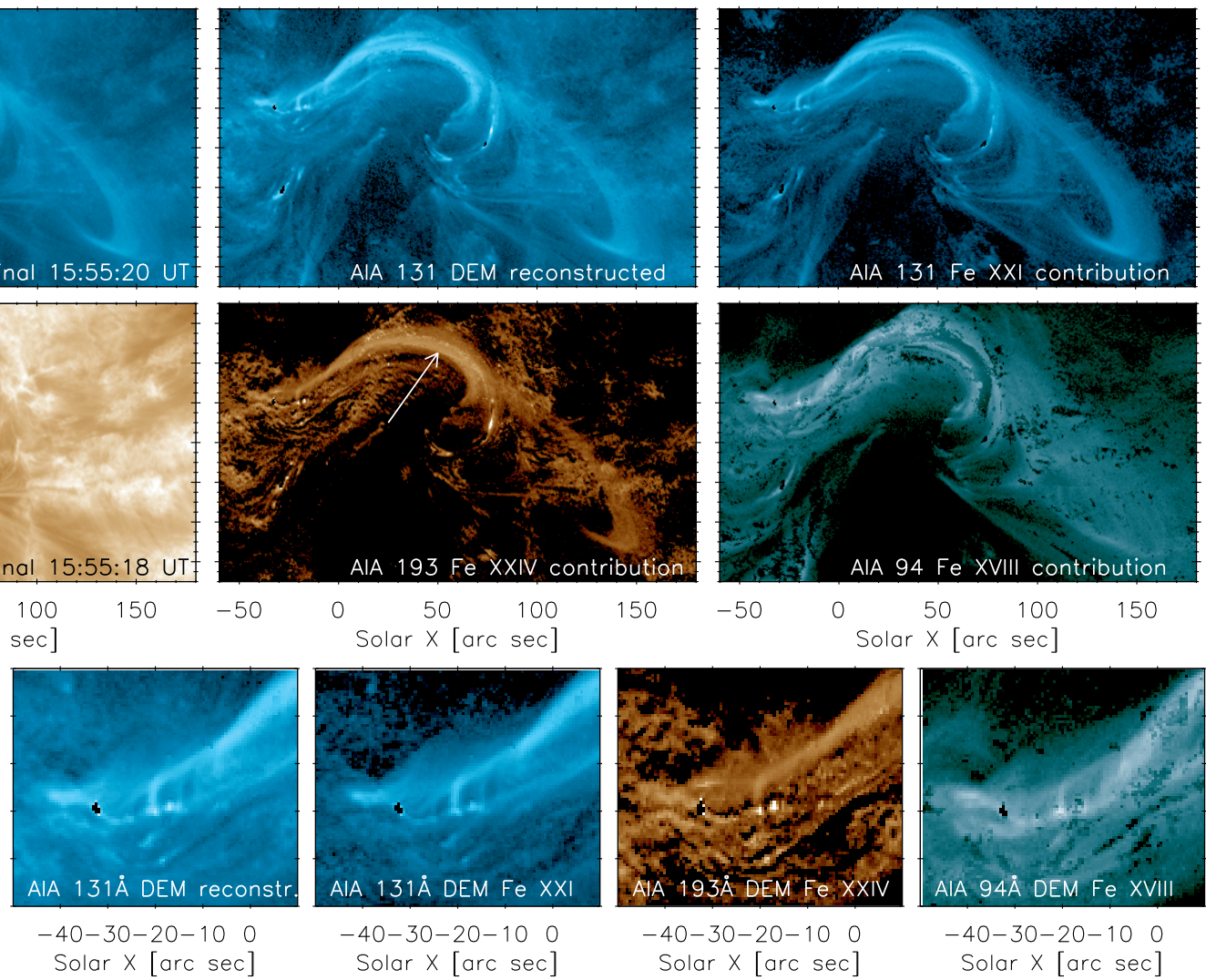

Figure 14. DEM reconstruction of the AIA data at 15:55 UT and the main contributions to individual AIA channels. Top, from left to right: AIA $131 \AA$ observed, AIA $131 \AA$ reconstructed from DEM, and Fe XXI contribution to AIA 131A. Middle, from left to right: AIA 193 observed, contribution from Fe XXIV to AIA $193 \AA$ channel, and contribution from Fe XVIII to AIA 94A channel. The box in the top left image indicates the zoom-in region shown in the bottom row. White Arrow denotes flare loops emitting in Fe XXIV. Bottom: Close-up on the footpoints of the apparently slipping flare loops. The field of view corresponds to that of Fig. 8 . All intensities are scaled logarithmically, with units of $D N s^{-1} \mathrm{px}^{-1}$. The scale is the same as in Fig.8. with AIA $193 \AA$ having the same scale as AIA $171 \AA$ in Fig. 8

which is the dominant contributor to the $131 \AA$ bandpass. In our case, Fe XXI contributes up to $\approx 50-85 \%$ of the observed flare emission in the $131 \AA$ channel. Weak Fe XXIV emission is also present in the $193 \AA$ bandpass (Fig. 14, middle row, center.) This Fe XXIV emission contributes of about $\approx 45 \%$ to the observed flare loops in the $193 \AA$ image (white arrow in Fig. 14). We note that these loops are weak in the $193 \AA$ image and can be discerned only outside of areas of strong moss emisson that dominate the observed $193 \AA$ morphology.

We note that there is some spurious contribution in the moss areas in the recovered Fe XXI and Fe XXIV intensities. However, this emission is typically $\approx 30$ times weaker compared to the observed signal in the $193 \AA$ channel. The recovered DEM at these locations exhibit a weak secondary peak at $\log (T / K)=7.2$ characterized again by large errors, and therefore uncertain.

In summary, the DEM reconstruction confirms that the slipping loops consist dominantly of flare plasma with temperatures up to $\log (T / K)=7.1-7.3$.

\section{NUMERICAL SIMULATION OF A FLUX ROPE EXPANSION AND ASSOCIATED SLIPPING RECONNECTION}

The mechanisms of solar flares and the associated formation of magnetic structures can be well reproduced with numerical models. In the following, we exploit the
3D MHD simulation performed earlier by Aulanier et al. (2012), recreating the evolution of a flux rope expansion during an eruptive flare. This simulation exhibits naturally occurring slipping magnetic reconnection as a result of the evolution of the model. This slipping reconnection builds both the flare loop arcade and the erupting flux rope (Aulanier et al. 2012, Janvier et al. 2013), which, as we will show in this section, compares well with the observations in qualitative terms. Although the simulation was not designed to fit any specific event, it is well suited for this particular flare (described in Sect. 2), as the photospheric magnetic field contains flux asymmetry, and geometrical comparison with the observations can be easily achieved by appropriate rotation of the simulation box.

\subsection{Description of the numerical simulation}

The initial conditions of the model are dynamically built so that the whole magnetic structure is torusunstable. The details of the physical ingredients needed to build such conditions are described in Aulanier et al. (2010). The development of the torus instability leads to the upward expansion of the flux rope core, as well as the formation of a thin current layer where reconnection takes place. The reconnected field lines are of two types: they either further add to the envelope of the flux rope or they form the flare loops, as described in Aulanier et al. 
(2012) and Janvier et al. (2013). The numerical simulation is non-dimensionalized, and extends over a time period of $46 t_{A}$, where $t_{A}$ represents the Alfvén time, i.e., the travel time for a distance $d=1$ at the Alfvén speed $c_{A}=1$.

The region modelled in the present numerical simulation has similar features as that of the AR 11520 where the X1.4 flare was observed (Sect. 2). First, the asymmetry of the magnetic polarities is reproduced, with a $27 \%$ flux imbalance favouring the positive polarity in the photosphere. This asymmetry reflects the stronger, leading positive polarity and the weaker, trailing negative polarity of AR 11520 (Fig. 1). The numerical simulation also reproduces well the shape of the sigmoid as seen in the $94 \AA$ filter at the beginning of the flare (Fig. 1). This sigmoid, present at $t=15: 00 \mathrm{UT}$, contains

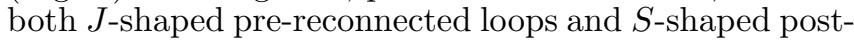
reconnected loops as seen from the top, as is shown in the top right image of Fig. 17, where field lines are drawn at one time in the simulation.

Lack of null points and separatrices in the simulation implies that reconnection takes place in QSLs. Field lines then undergo a succession of reconnection processes as they cross the evolving QSLs, resulting in an apparent slipping motion. The details of such a mechanism have been thoroughly investigated in Janvier et al. (2013).

\subsection{QSL structural evolution}

Quasi-separatrix layers, that correspond to a strong distortion of the magnetic field, are for the present simulation calculated with the TOPOTR routine (Démoulin et al. 1996) that integrates all the field lines of the volume from a fixed position in one polarity to its counterpart in the other polarity. TOPOTR then measures the squashing degree $Q$, that is a quantification of the magnetic field distortion (Pariat \& Démoulin 2012), and that defines QSLs for $Q>2$. 2D maps of QSLs can then be drawn anywhere in the volume, representing e.g. the QSL footpoints with a cut at the photosphere, or the hyperbolic flux tube (HFT) with a vertical cut into the volume which corresponds to the central part of the QSL volume.

An example of these QSL footpoints at the photosphere $(z=0)$ can be found in Fig. 15 , where we have plotted the contour plot of $\log (Q)$ at three different times during the flux rope expansion of the present numerical simulation. The colour table indicates the strongest magnetic field gradients corresponding to $\log (Q) \geqq 8$ in red, and weaker gradients with $\log (Q) \rightarrow 0$ in blue. The regions in white correspond to areas where magnetic field lines are open, making the calculation of $Q$ impossible (see also Janvier et al. 2013). The magnetic polarities in the photosphere are indicated by contour plots, with purple and cyan for the positive and negative polarities, respectively.

We note that since QSLs correspond to regions of high magnetic field distortion, they are expected to be formed near strong current density locations, as was demonstrated in Savcheva et al. (2012), Gekelman et al. (2012) and Janvier et al. (2013), although there is not necessarily a one-to-one correspondence (Wilmot-Smith et al. 2009). Then, the evolution of QSLs is very similar to that of flare ribbons, as is described in Sec.2 of Janvier et al. (2013).

At $t=5 t_{A}$, two very thin QSL structures exist (Fig.
15. left, red color). Both are QSLs consisting of a straight part and a hook. Note that similarly to the magnetic polarity asymmetry, there is an asymmetry in the shape of those QSLs, most pronounced for the hooks. The QSL in the stronger positive polarity has a rounder hook, and is localized very near the center of the polarity. In contrast to this, the hook of the QSL in the weaker negative polarity extends toward negative values and is much broader, while the tip of the hook remains located near the centre of the polarity. The straight part of both QSLs is close to the polarity inversion line (PIL) indicated in yellow colour.

As time advances, two evolutions can be seen. First, the QSLs move away from each other, i.e., their straight parts move away from the PIL. This motion resembles the flare ribbon motion, as was suggested by Janvier et al. (2013) and as can be seen here by comparison with observations. Indeed, the distance between flare ribbons NR and PR in $304 \AA$ filter (Fig, 3) increases over time, suggesting the separation of flare ribbons as is commonly observed during eruptive flare events.

Secondly, both QSL hooks become rounder with time. This is straightforward with the QSL in the negative polarity, as it extends toward negative $y$-direction in the simulation (Fig. 15) and becomes rounder. The orientation of this extension corresponds to the east direction on the surface of the Sun, and is similar to the evolution

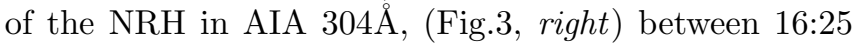
and 17:00 UT. However, the observed NRH does not become rounder, instead it elongates in the south direction and remains narrow, as was described in Sect. 2.1. This is because the large-scale magnetic configuration of the AR 11520 and 11521 is much more complex than in the simulation. The presence of surrounding magnetic fields on the Sun implies the existence of numerous large-scale QSLs that prevent the extension of the flare-associated QSLs (Chen et al. 2012). The broadening QSL is then squeezed by the surrounding structures, therefore explaining the unidirectional elongation in the observations instead of the isotropic broadening seen in the simulation.

In the simulation, the QSL hook in the positive polarity does not extend much and while it becomes rounder, it remains located near the center of the polarity. It is however difficult to compare the evolution of this QSL hook in the positive polarity with the observations. In Fig.3, the PRH is in fact an elongated ribbon, whose structure could be more complicated than in the simulation due to presence of other magnetic polarities, i.e., AR 11521.

For completeness, we note that at $t=45 t_{A}$ another QSL is present in the negative polarity in the simulation. Its straight part lies very close to the PIL, and the hook very close to the previously described QSL. This QSL is associated with a bald patch corresponding to field lines tangent to the surface. The bald patch does not play any role in the reconnection of field lines in the simulation. Its description will then be omitted in the further paragraphs.

The evolution of the QSLs is associated with the reconnection process leading to the formation of new pairs of reconnected field lines, including the flux rope envelope. This process is detailed in the following. 

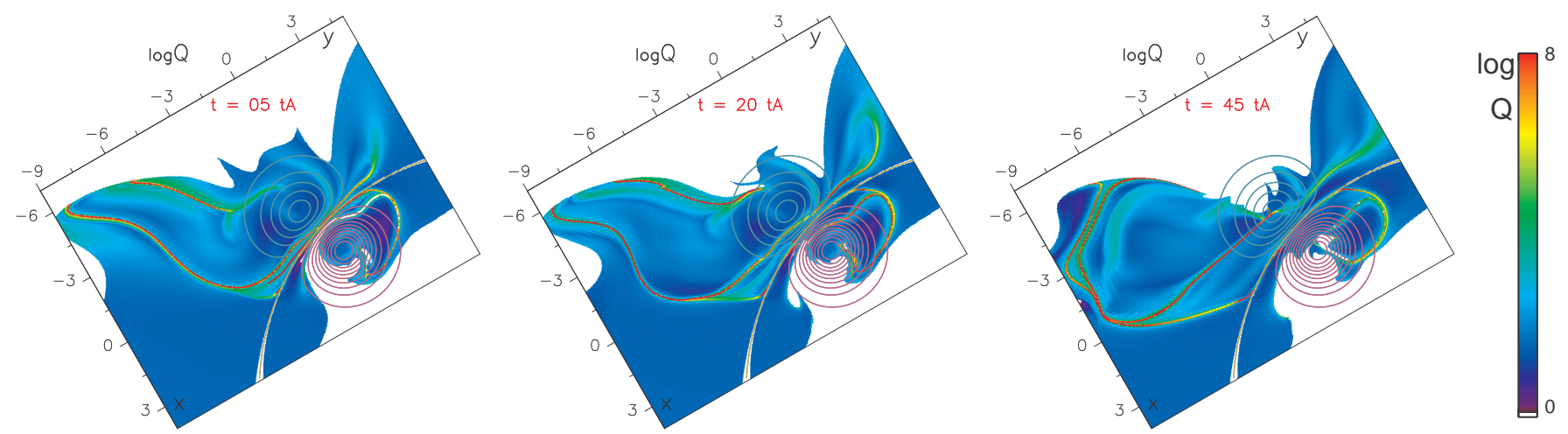

Figure 15. Evolution of the photospheric footpoints of the QSLs in the torus-unstable flux rope simulation with increasing time, showing the structural chages of the QSLs, as well as changes in their magnitude. Individual colors depict the value of $\log (Q)$ of the squashing factor $Q$ at the photosphere. $Q$ is plotted only in areas where the magnetic field lines are locally closed within the computational box. Pink and cyan contours denote the positive and negative magnetic polarities in the model, with the positive one being stronger than the negative one.

\subsection{Slipping motion and associated kernel brightening}

In the simulation, the upward expansion of the flux rope during its ejection creates a very thin current layer where reconnection occurs. This current layer is associated with a HFT, as described in Fig. 2 in Janvier et al. (2013). The field lines passing through the HFT undergo multiple reconnections, and this effect is seen as an apparent slippage of the field lines. In order to investigate this fast apparent motion in detail, the numerical data have been output from the simulation at a time cadence of less than one Alfvén time.

Figure 16 shows a set of selected field lines that are evolving from $t=20$ to $t=20.7 t_{A}$. We picked these times since the magnetic field lines resemble the shape of the flare loops (Fig. 3). The photospheric surface at $z=0$ is shaded according to the photospheric current density $j_{z}$, with the magnetic polarities indicated by pink and cyan contour plots. The field lines are integrated from their fixed footpoints anchored in the positive polarity close to the tip of the QSL hook. This hook is apparent in both the QSL trace (Fig.15, middle) and the $j_{z}$ structures. With advancing time, the connectivity of the field lines changes. For example, the blue field line is anchored in the negative polarity near the PIL at $t=20 t_{A}$, while at $t=20.2 t_{A}$ it extends beyond the field of view of the simulation box, and at $t=20.4 t_{A}$ is connected to the tip of the hook of strong $j_{z}$, similar in shape to the QSL hook in the negative polarity. This motion occurs similarly for the other field lines so that at $t=20.7 t_{A}$, they are all connected in the negative polarity near the tip of the QSL or $j_{z}$ hook. The continuity of this motion can be seen in the online Movie 7 .

This motion can in principle be observed with SDO/AIA imaging by two means. First, the loops themselves can be seen to be moving, as is the case for their NR footpoints discussed in Sects. 2.2.1 2.2.3 (also Figs. 6. 8. 10). Second, the field line footpoints can be seen to be moving as are the ribbon brightenings. These correspond to chromospheric or transition region emission due to the impact of energetic particles accelerated in the reconnection site. In practice, both are observed at the same time (Sect. 2.2).

We note that in the simulation, the fast motion of field lines is only an apparent motion due to the diffusion of magnetic field in the current layer, not necessarily as- sociated with motion of plasma dragged by the moving field lines. This is because the time scale needed to fill the field lines by chromospheric evaporation is generally much larger than the time scale for multiple reconnections to take place in the current sheet. However, if reconnection were to happen on a time scale that is comparable with evaporation-field line filling, then the slipping motion of field lines could be identical with that of the flare loops. Note that the AIA instrument is able to observe only the portions of the field lines filled with highdensity plasma at temperatures given by its temperature response (Fig. 2). Therefore, the coherent, apparently moving loops, reported in Sect. 2.2 do not neccessarily lie on a single, co-moving (slipping) magnetic field line. Rather, the apparent slipping motion of these loops is an illusion created by the apparent slipping motion of the magnetic field itself, coupled with the plasma thermal response.

We also note that the speed of the slipping motion along the QSL is not uniform. This can be inferred also from Fig 16. At $t=20 t_{A}$ and $t=20.4 t_{A}$, field lines are only slightly moving in the straight part of the QSL, while their motion becomes much faster in the hook, as can be seen by comparing $t=20.4 t_{A}$ with $t=20.7 t_{A}$. This slipping velocity profile has been investigated in detail in Sect. 3 of Janvier et al. (2013), where a peak in the velocity profile was found near the hook of the QSL. Smaller velocities were found at the beginning and at the end of the slipping motion, corresponding to the straight part of the $J$-shape and tip part of the hook. The peak in the velocity profile can be explained by the fact that the reconnecting field lines are passing through the HFT, where $Q$ is the highest, and the changes in magnetic connectivity the most drastic.

This change of slipping motion speed can also be seen in the observations (Sect. 2). As seen in Figs. 6, 8 and 10 one can see different sets of loops slipping at different times during the flare, yet their footpoints are mostly located in the straight part of the NR (Fig. 3). These slipping loops can be seen in this location because the field line slipping motion is slow, leaving enough time for filling the field lines via chromospheric evaporation. It is then possible to measure the velocity of this motion with stackplots along different cuts (Figs. 7, 9, and 11). Similarly, a bright loop appears at the tip of the NRH at 

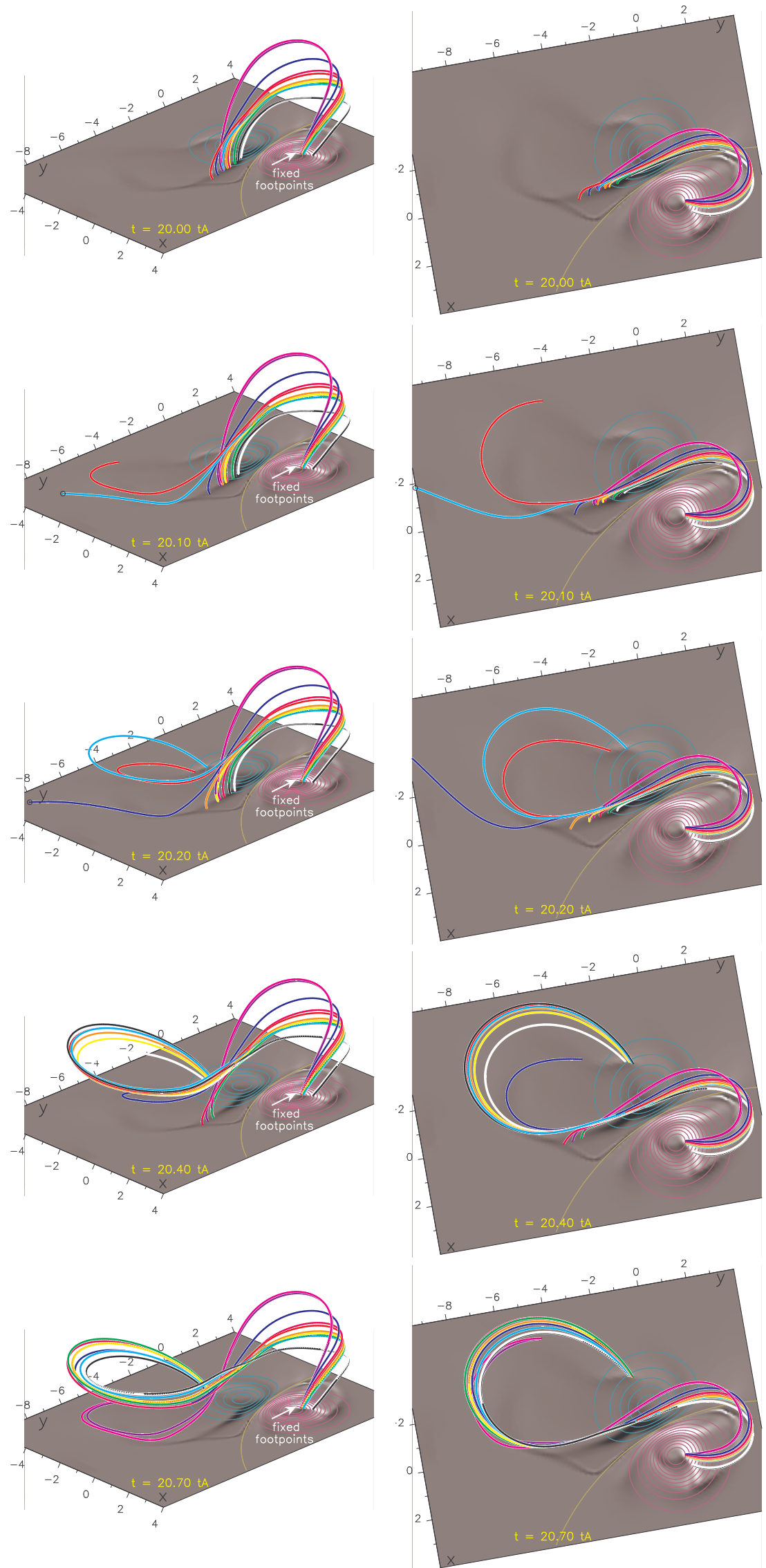

Figure 16. Images from different times of the flux rope simulation showing the evolution of the field lines undergoing slipping reconnection. Right: top view; left: side view. With increasing time, the field lines move from the central part of the J-shaped current region (dark area along the PIL) lying along the QSL to the tip of the hook, and finally become a part of the flux rope envelope. 
16:10:08 UT (Arrow 3 in Fig 3). This loop can be associated with a slipping set of field lines found at 15:55:08 UT.

Contrary to flare loops, kernel brightenings are much more visible moving along the ribbons, as can be seen in the NRH in the online Movie 2 and also in the Fig. 3 (right column). However, this kernel motion is not continuous but rather scattered, although the associated reconnection process should be continuous. This can be explained in terms of energy deposition along the QSL footpoints. The energy from the reconnection process is deposited at the chromosphere thanks to the energetic particles accelerated at the reconnection site. When the field lines are moving slowly, the energy is deposited in a small area of the QSL footpoint, so that the resulting chromospheric or EUV emission due to evaporation is large. In contrast, when the field lines move fast, the energy deposited per area is small, so that the kernel brightening will be weaker or even practically invisible. This is illustrated in Fig 16 where the footpoints of the field lines are seen to jump from the straight part of the QSL to the tip of the hook. This jump indicates that the change of connectivity is much more important in the hook. Therefore, the deposition of energy in the hook should be very low. At 16:25 UT, the intensity of the $\mathrm{NRH}$ is indeed slightly lower than the straight part of the NR, or the NRH hook (Fig. 3, fifth column).

The direction of the propagation of slipping loops during the observed flare is also consistent with the simulation. In the numerical model, the footpoints of the reconnecting field lines move along the QSL footpoint (Fig,16) toward the tip of the hook. This propagation direction is clearly seen in Figs. 6, 8, and 10.

\subsection{Evolution of the expanding flux rope}

The slipping motion of field lines, as discussed above, occurs on a very short time scale, typically within one Alfvén time. When the magnetic field lines reconnect, they either add to flare arcade or they contribute to the flux rope envelope. We are now concerned with the latter process.

As time passes, the reconnection process feeds the flux rope with twisted field lines surrounding its core, leading to the continuous growth of the expanding flux rope (e.g., Fig.5 in Aulanier et al. 2012). This process, reproduced in the present 3D simulation, is similar to that depicted in the CSHKP model where twisted field lines construct the envelope of the flux rope. This growth of the flux rope is related with the motion of QSLs within the volume. Similar to separatrices in a quadripolar configuration, QSLs move away from the reconnection region with increasing time (Sect. 4.2). The moving QSLs swipe the field lines that subsequently reconnect as they are passing in the QSLs.

Therefore, the footpoints of the field lines constituting the flux rope, that have therefore already reconnected, are located inside the two hooks of the QSL footpoints in the negative and positive polarities, as shown in Fig 15 . Pre-reconnected field lines that will become a part of the flux rope are in contrast situated on the periphery, i.e., outside the hook. The shape of the hooks therefore gives a good indication on the localization of the flux rope footpoints as well as its growth with time: the rounder and bigger the hooks become indicates how "big" the flux rope becomes as well. In summary, the envelope of the flux rope increases from the peripheral region by feeding the flux rope as time goes by.

The evolution of the flux rope envelope is shown in Fig 17. There, we have represented a set of field lines in pink representing the unstable flux rope core present from the beginning of the simulation. Another set of field lines, reconnecting between $t=20 t_{A}$ and $t=27 t_{A}$, is shown in blue. These lines are actually the same set of field lines that are slipping from $t=20$ to $t=20.7 t_{A}$ in Fig.16. Finally, green represent field lines reconnecting at a later time, between $t=27$ to $t=34 t_{A}$. We note that field lines reconnecting at later times surround the field lines already reconnected, i.e., the green lines are winding around both the pink and blue ones. Note also that as the flux rope expands, the whole structure becomes increasingly stretched, leading to almost vertical field lines near their footpoints at the photosphere, as is apparent from the side view in Fig. 17 (left column) at $t=41 t_{A}$.

The top view shown in the right panel of Fig 17 can be compared directly with observations of the flare (Fig 3 . arrows 2 and 3; Fig. 5). These long, hot loops appearing on the sides of the active region are added to the whole flux rope structure by the periphery and constitute its envelope (Fig. 5). The flux rope core itself is not visible.

The mechanism behind these erupting loops can be understood when looking at the top view (right panel) of Fig 17. The flux rope tends to erupt in a privileged direction (green lines in Fig. 17). In the simulation, this privileged direction is north-east. In the observations, the erupting loops also have a preferential direction, although this is south-west (Arrow 2 in Fig. 3). The existence of a privileged direction arises due to the asymmetry of the flux rope expansion, but can be constrained by the surrounding magnetic field, as observed here and described in Sect. 2. Moreover, the green field lines are seen to erupt in the model from $t=34$ to $t=41 t_{A}$. This shows that only parts of the flux rope erupt at different times, explaining the appearance of erupting loops on the west (arrow 2) at $t=15: 40: 08$ UT before those on the east (arrow 3) at $t=16: 10: 08$ UT as shown in Fig.3. Therefore, the classical approach to flux rope ejection, as described in McKenzie \& Canfield (2008) is not complete: the flux rope is continuously fed by on-going reconnection, leading to different sets of field lines constituting the flux rope envelope having their own ejection dynamics.

\section{RADIO OBSERVATIONS AND INTERPRETATION}

To gain more insight into the nature of the slipping reconnection process, we analysed the available radio data for the flare. These also complement the EUV observations from SDO/AIA.

At about 15:00 UT, radio burst activity started as a noise storm in the $200-500 \mathrm{MHz}$ range (Fig. 18, first row). The noise storm was observed by the Callisto instrument (Benz et al. 2009; Monstein 2013) in Trieste and consisted of a group of narrowband bursts resembling narrowband type III bursts. Some of the individual bursts exhibited drifts towards lower and some to higher frequencies. The typical frequency drifts of these bursts, which are about $20 \mathrm{MHz} \mathrm{s}^{-1}$, are more than an order of magnitude smaller than the typical mean frequency drift 

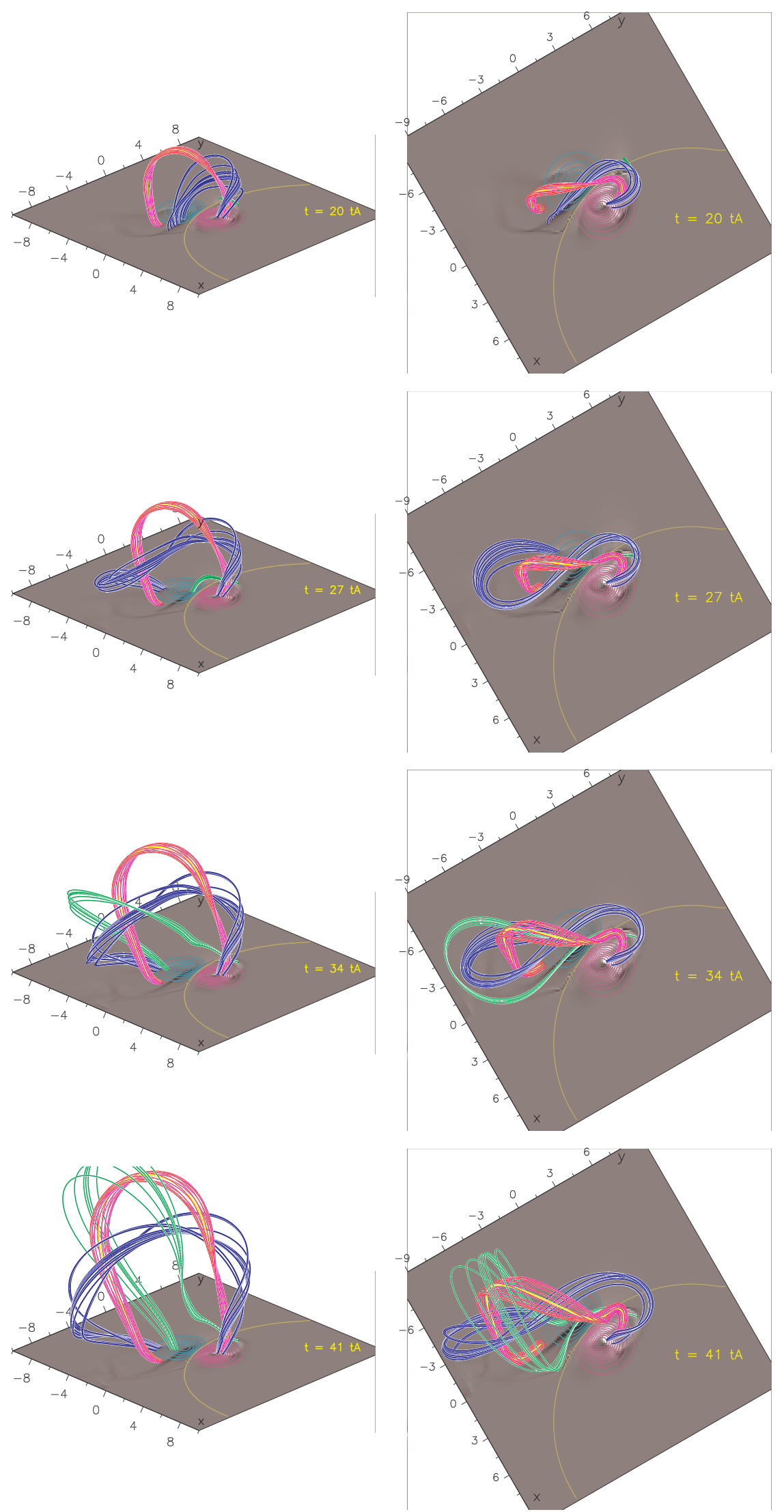

Figure 17. Evolution of the flux rope with increasing time. Right: top view; left: side view. The initial flux rope is indicated in pink, while successively reconnecting field lines are indicated in blue (reconnection from $t=20 t_{\mathrm{A}}$ ) and green $\left(\right.$ reconnection from $\left.t=27 t_{\mathrm{A}}\right)$. The final image shows the expansion of the whole structure and the envelope successively formed by magnetic lines reconnecting at different times. 

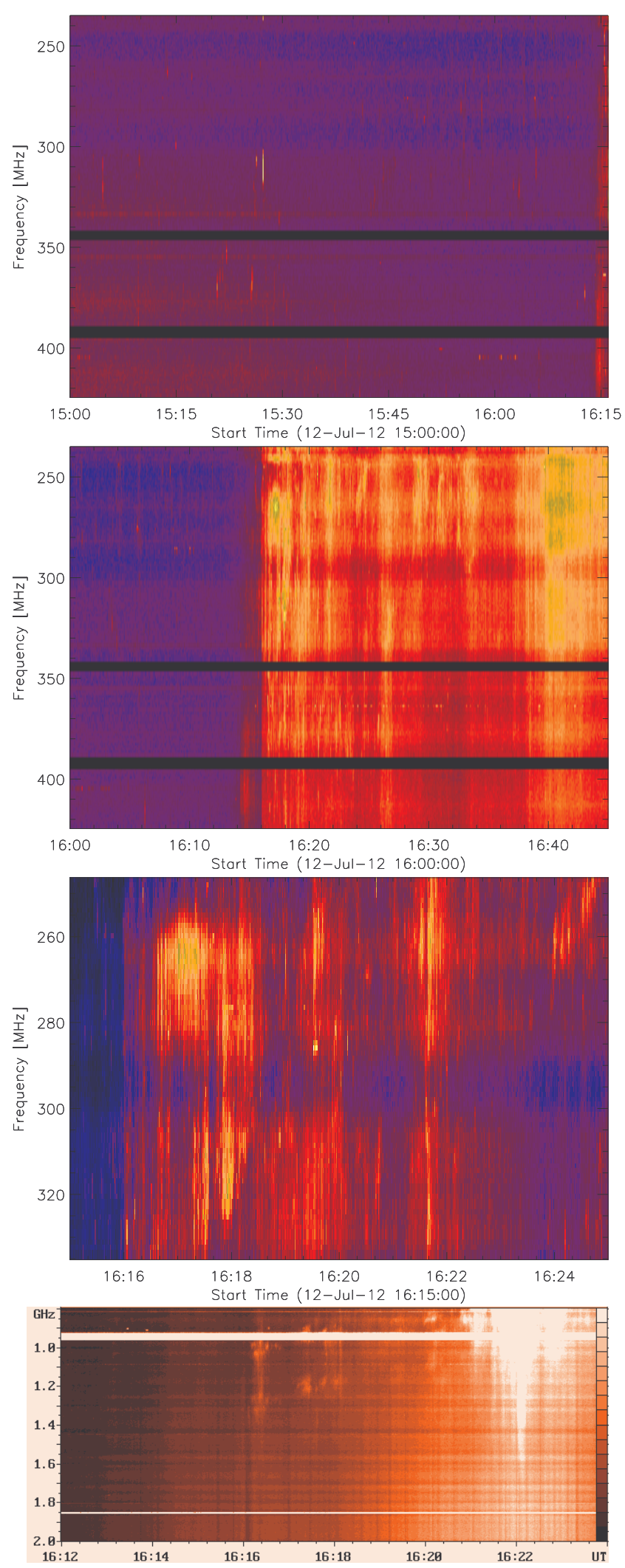

Figure 18. Radio spectra of the flare observed by the Callisto instrument in Trieste (first to third row) and by the radio spectrograph at the Ondřejov observatory (bottom). First and second rows: Spectra from 15:00 UT to 16:49 UT, capturing the early phase characterized by the noise storm, and the impulsive phase beginning at 16:16 UT. Third and bottom rows: Detail of the DPSs at the start of the impulsive phase around 16:16 UT. of the type III bursts, which is about $360 \mathrm{MHz} \mathrm{s}^{-1}$ Alvarez \& Haddock 1973). This means that the velocity of the electron beams generating the noise storm bursts is more than an order of magnitude slower than for type III radio bursts, which is about $c / 3$, where $c$ is the speed of light.

The noise storm lasted until 16:16 UT, when the strong radio flare started. The beginning of the strong radio flare is marked with two drifting pulsation structures (DPSs, e.g., Karlický et al. 2002, Karlický 2004) occurring during 16:16-16:21 UT: one starting at $1.3 \mathrm{GHz}$ and the other one at $1.0 \mathrm{GHz}$ (Fig. 18, bottom row). Since these frequencies are not in harmonic relationship, this indicates spatially separated plasmoids. Both these DPSs drifted towards lower frequencies with the frequency drift $\mathrm{d} f / \mathrm{d} t=-0.8 \mathrm{MHz} \mathrm{s}^{-1}$. Based on the timing, we identify them with the eruption of the series of long S-shaped loops observed by AIA (Fig. 5). Note also that at about 16:16:30 UT, several DPSs also appeared in the $250-300 \mathrm{MHz}$ range (Fig. 18, third row), c.f. Karlický (2004), indicating a range of erupting structures with different densities.

At 16:16:30 UT, broadband radio continuum was registered in the range of $1-5 \mathrm{GHz}$ simultaneously with the DPSs. The $1-5 \mathrm{GHz}$ radio emission peaked at about 16:22 UT. After a short decrease at about of 16:24 UT the radio emission increased to its main maximum at 16:26 UT. At the lower frequencies $(200-500 \mathrm{MHz})$, the main maximum occurred at about 16:40 UT.

The bursty nature of the radio emission in both the noise storm and the DPSs shows that the reconnection during the flare is intermittent and that the energy release leading to plasma heating is not uniformly distributed in time or space. This is already hinted at by the EUV observations from AIA (Sect. 2), which showed discrete, apparently moving features in all filters, rather than a continuous, near-uniform heating over the spatial locations (ribbons) where the slipping motion of the field line footpoints is occurring. Furthermore, presence of the DPSs during the impulsive phase indicates (1) presence of a current sheet and its fragmentation, (2) enhancement of the reconnection rate (Kliem et al. 2000, Bárta et al. 2008,2011 ) at 16:16 UT, and that (3) the fast reconnection is not of the Petschek (1964) type, but turbulent with plasmoids in 3D (Daughton et al. 2011a b). Note that the 3D MHD model of Aulanier et al. (2012) does not have sufficient spatial resolution to resolve the individual small-scale processes within the current sheet. Furthermore, since the model is pressureless, it does not include spatial and temporal distributions of plasma heating and particle acceleration. Therefore, the radio data give an important complementary information to the flare physics treated by the 3D MHD model.

\section{SUMMARY AND DISCUSSION}

We have presented observations of an eruptive X1.4 long-duration flare that occurred on July 12, 2012 in active region 11520 , which is a part of an active region complex. The observations were compared to the 3D MHD "standard solar flare model" of Aulanier et al. (2012). The model qualitatively allows for an explanation of the observed apparent slipping motion of both the flare and erupting loops in terms of a torus-unstable erupting flux rope that is fed continually by the slipping magnetic re- 
connection.

The flare itself is preceded by a brightening of several loop systems located in active region 11521 . Using magnetic field extrapolations, we found that one of these loop systems shares the footpoint of a quasi-separatrix layer corresponding to the positive-polarity flare ribbon PR.

The flare starts with the appearance of a highly sheared flare loop in AIA $131 \AA$, outlying an active region filament F1 seen in $304 \AA$. This flare loop appears in a pre-existing coronal sigmoid. It subsequently develops into an arcade of flare loops, with individual loops exhibiting apparent slipping motion. In the early stages of the flare, there are several episodes when the slipping motion is clearly visible. This apparent slipping motion is of the order of several tens of $\mathrm{km} \mathrm{s}^{-1}$ and is most pronounced in the flare loop footpoints located in the ribbon in the trailing negative polarities. Transition region emission from the loop footpoints is clearly identifiable in $171 \AA$, $304 \AA$ and $1600 \AA$ from the beginning of the flare. These footpoints represent the first signature of the ribbon and subsequent local brightenings along the developing ribbon.

A number of the flare loops observed in $131 \AA$ expand in the SW direction and subsequently erupt. These loops connect both flare ribbons and contain a series of faint, S-shaped non-potential loops more than $250^{\prime \prime}$ long. The footpoints of the erupting loops are seen to slip along the extended hook of the positive-polarity ribbon with a velocity of approximately $136 \mathrm{~km} \mathrm{~s}^{-1}$. A CME is subsequently observed by the STEREO spacecrafts.

The DEM analysis method of Hannah \& Kontar (2012) was applied to each AIA pixel in a limited field of view. It confirms that both the apparently slipping and erupting flare loops emit strongly in Fe XXI, originating around $10 \mathrm{MK}$. This emission is seen in the $131 \AA$ channel. Portions of these loops are also visible in the $94 \AA$ or $193 \AA$ channels, dominated by contributions from Fe XVIII, Ca XVII and Fe XXIV. A DEM analysis leads to an estimation of these contributions. We show that the flare loops do indeed emit in Fe XXIV. This emission is, except for a portion of the flare loops above the inversion line, obscured by much stronger Fe XII emission coming from the moss and warm coronal loops.

The observations have been qualitatively explained by a 3D pressureless MHD simulation of Aulanier et al. (2012) and Janvier et al. (2013). In this simulation, a torus-unstable flux rope is located within an active region exhibiting flux imbalance similarly to our observations. The simulation does not contain null points or separatrices, but the presence of quasi-separatrix layers leads to a slipping reconnection regime. That is, the field lines with one fixed footpoint in a QSL exhibit an apparent slipping motion of the footpoint in the conjugate QSL. The direction of the slipping predicted by the model is consistent with the observations. The difference between the simulated and observed velocities may be caused by the difference between the timescales of the slipping motion and chromospheric evaporation, as the flare loops must first be filled with heated plasma in order to be observable. Alternatively, the slipping reconnection during the flare can happen in thicker QSLs, involving sub-Alfvénic flare loop slipping motion, as suggested in Aulanier et al. (2007), instead of apparent super-Alfvénic motion in thin QSLs as in Janvier et al. (2013).
The QSL footpoints in the simulation are in the shape of a hook, with a straight portion in the strong photospheric magnetic field, and a curved hook portion located further away. The simulated slipping motion is faster in the hook, in agreement with the observations. During the simulation, the hook evolves and becomes rounder. The presence of a hook and its evolution is reflected in the observations, with the exception that large-scale magnetic field constricts the negative-polarity hook and does not allow it to become round. Instead, the hook is deformed because of the presence of a large-scale QSL and extends more than $100^{\prime \prime}$ to the south. The EUV intensity of the ribbon hook is lower than that of the straight portion of the ribbon, which can be understood in terms of energy deposition. The slipping motion is faster in the hook than in the straight part of the QSL in both the observations and the simulation, resulting in a lower amount of energy per unit time and area available for chromospheric evaporation.

The flux rope in the simulation is unstable and expands, which leads to its eruption. As the reconnection proceeds, the flux rope is fed with newly reconnected field lines that participate in the eruption. The flux rope envelope is observed by the AIA as long, S-shaped erupting hot loops. The erupting flux rope has a preferential direction in both the model and observations. In the observations this direction is modified by the large-scale magnetic field not present in the model.

An interesting feature of the flare is that despite the presence of an erupting flux rope and a flare arcade, the filament F1 remains unperturbed during the entire flare. This suggests that the real flare configurations may be complicated by the presence of another, filament-related flux rope that does not evolve with the rest of the magnetic configuration, in particular the overlying sigmoid. We note that although there are magnetic dips close to the photosphere in the simulation (Aulanier et al. 2012, Fig. 6 therein), these are part of the sigmoid and not of any additional flux rope. This additional flux rope must then be connected to the topological complexity of real solar magnetic fields, as opposed to the simplified modelled ones. We also note that such tightly-packed flux-ropes constituting active-region filaments have indeed been recently reported by Kuckein et al. (2012) and Yelles Chaouche et al. (2012).

The apparent motion of EUV loops in early stages of the flare, interpreted by the slipping reconnection, was associated with the noise storm in the metric radio range. The noise storm ended at 16:16 UT with the appearance of the dm-drifting pulsation structures, indicating plasmoid formation within the flare current sheet and their subsequent ejection. This marks the enhancement of the reconnection rate and the impulsive phase of the flare, as evidenced by radio bursts observed in very broad range of radio frequencies. Note that at this instant the long S-shaped loop erupted and the GOES X-ray flux rapidly increased.

In summary, we have shown that the apparent slipping motion as a result of slipping reconnection, is indeed occurring during eruptive flares. This motion is a typical feature of the "standard solar flare model in 3D", which allows for a consistent explanation of many of the individual magnetically-controlled phenomena during the eruptive flares. It also shows that null-points and true 
separatrices are not required for the eruptive flares to occur. Radio data indicate that the slipping reconnection is also associated with intermittent particle acceleration and plasmoid formation.

AIA data are courtesy of NASA/SDO and the AIA science team. JD acknowledges support from the Royal Society via the Newton Fellowship Programme. This work was supported by Scientific Grant Agency, VEGA, Slovakia, Grant No. 1/0240/11. GDZ acknowledges support from STFC (UK) via the Advanced Fellowships Programme. HEM also acknowledges support from STFC. The work of MK was supported by the Grant No. 209/12/0103 of the Grant Agency of the Czech Republic. CHIANTI is a collaborative project involving the NRL (USA), RAL (UK), MSSL (UK), the Universities of Florence (Italy) and Cambridge (UK), and George Mason University (USA).

\section{REFERENCES}

Alexander, C. E., Walsh, R. W., Régnier, S., et al. 2013, ApJ, 775, L32

Alissandrakis, C. E. 1981, A\&A, 100, 197

Alvarez, H., \& Haddock, F. T. 1973, Sol. Phys., 29, 197

Amari, T., Luciani, J. F., Mikic, Z., \& Linker, J. 2000, ApJ, 529, L49

Andretta, V., Del Zanna, G., \& Jordan, S. D. 2003, A\&A, 400, 737

Antiochos, S. K., DeVore, C. R., \& Klimchuk, J. A. 1999, ApJ, 510,485

Aschwanden, M. J., \& Boerner, P. 2011, ApJ, 732, 81

Asplund, M., Grevesse, N., Sauval, A. J., \& Scott, P. 2009, ARA\&A, 47, 481

Aulanier, G., Démoulin, P., \& Grappin, R. 2005, A\&A, 430, 1067

Aulanier, G., Janvier, M., \& Schmieder, B. 2012, A\&A, 543, A110

Aulanier, G., Pariat, E., Démoulin, P., \& DeVore, C. R. 2006, Sol. Phys., 238, 347

Aulanier, G., Török, T., Démoulin, P., \& DeLuca, E. E. 2010, ApJ, 708, 314

Aulanier, G., Golub, L., DeLuca, E. E., et al. 2007, Science, 318, 1588

Aurass, H., Mann, G., Zlobec, P., \& Karlický, M. 2011, ApJ, 730, 57

Bárta, M., Büchner, J., Karlický, M., \& Skála, J. 2011, ApJ, 737, 24

Bárta, M., Vršnak, B., \& Karlický, M. 2008, A\&A, 477, 649

Benz, A. O., Monstein, C., Meyer, H., et al. 2009, Earth Moon and Planets, 104, 277

Boerner, P., Edwards, C., Lemen, J., et al. 2012, Sol. Phys., 275, 41

Brosius, J. W. 2013, ApJ, 762, 133

Brosius, J. W., \& Holman, G. D. 2010, ApJ, 720, 1472

Carmichael, H. 1964, NASA Special Publication, 50, 451

Chandra, R., Schmieder, B., Aulanier, G., \& Malherbe, J. M. 2009, Sol. Phys., 258, 53

Chen, P. F., Su, J. T., Guo, Y., \& Deng, Y. Y. 2012, Chi. Sci. Bull., 57, 1393

Cheng, X., Zhang, J., Ding, M. D., Liu, Y., \& Poomvises, W. 2013, ApJ, 763, 43

Chifor, C., Mason, H. E., Tripathi, D., Isobe, H., \& Asai, A. 2006, A\&A, 458, 965

Craig, I. J. D., \& Brown, J. C. 1976, A\&A, 49, 239

Craig, I. J. D., \& Brown, J. C. 1986, Inverse problems in astronomy: A guide to inversion strategies for remotely sensed data (Adam Hilger, Ltd., 159 p.)

Daughton, W., Roytershteyn, V., \& Karimabadi, H. 2011a, in APS Meeting Abstracts, 9016P

Daughton, W., Roytershteyn, V., Karimabadi, H., et al. 2011b, in American Institute of Physics Conference Series, Vol. 1320, American Institute of Physics Conference Series, ed. D. Vassiliadis, S. F. Fung, X. Shao, I. A. Daglis, \& J. D. Huba, $144-159$ del Zanna, G. 1999, PhD thesis, , Univ. of Central Lancashire, (1999)

Del Zanna, G. 2013, A\&A, 558, A73

Del Zanna, G., Aulanier, G., Klein, K.-L., \& Török, T. 2011a, A\&A, 526, A137

Del Zanna, G., Mitra-Kraev, U., Bradshaw, S. J., Mason, H. E., \& Asai, A. 2011b, A\&A, 526, A1

Del Zanna, G., O'Dwyer, B., \& Mason, H. E. 2011c, A\&A, 535, A46

Démoulin, P., Bagala, L. G., Mandrini, C. H., Hénoux, J. C., \& Rovira, M. G. 1997, A\&A, 325, 305

Démoulin, P., Hénoux, J. C., Priest, E. R., \& Mandrini, C. H. 1996, A\&A, 308, 643

Démoulin, P., Priest, E. R., \& Lonie, D. P. 1996

J. Geophys. Res., 101, 7631

Dere, K. P., Brueckner, G. E., Howard, R. A., Michels, D. J., \& Delaboudiniere, J. P. 1999, ApJ, 516, 465

Dere, K. P., Landi, E., Mason, H. E., Monsignori Fossi, B. C., \& Young, P. R. 1997, A\&AS, 125, 149

Doschek, G. A., Warren, H. P., \& Young, P. R. 2013, ApJ, 767, 55

Elgaroy, O. 1977, Solar noise storms, 43

Fan, Y. 2012, ApJ, 758, 60

Fletcher, L., Dennis, B. R., Hudson, H. S., et al. 2011, Space Sci. Rev., 159, 19

Gary, G. A. 1989, ApJS, 69, 323

Gekelman, W., Lawrence, E., \& Van Compernolle, B. 2012, ApJ, 753,131

Graham, D. R., Fletcher, L., \& Hannah, I. G. 2011, A\&A, 532, A27

Green, L. M., \& Kliem, B. 2009, ApJ, 700, L83

Green, L. M., Kliem, B., \& Wallace, A. J. 2011, A\&A, 526, A2

Hannah, I. G., \& Kontar, E. P. 2012, A\&A, 539, A146

-. 2013, A\&A, 553, A10

Hirayama, T. 1974, Sol. Phys., 34, 323

Inglis, A. R., \& Gilbert, H. R. 2013, ArXiv e-prints

Janvier, M., Aulanier, G., Pariat, E., \& Démoulin, P. 2013, A\&A, 555, A77

Jiang, Y., Yang, J., Hong, J., Bi, Y., \& Zheng, R. 2011, ApJ, 738, 179

Judge, P. G., Hubeny, V., \& Brown, J. C. 1997, ApJ, 475, 275

Kane, S. R. 1974, in IAU Symposium, Vol. 57, Coronal

Disturbances, ed. G. A. Newkirk, 105-141

Karlický, M. 2004, A\&A, 417, 325

Karlický, M., \& Bárta, M. 2007, A\&A, 464, 735

—. 2011, ApJ, 733, 107

Karlický, M., Bárta, M., \& Nickeler, D. 2012, A\&A, 541, A86

Karlický, M., Bárta, M., \& Rybák, J. 2010, A\&A, 514, A28

Karlický, M., Fárník, F., \& Mészárosová, H. 2002, A\&A, 395, 677

Kliem, B., Karlický, M., \& Benz, A. O. 2000, A\&A, 360, 715

Kliem, B., Linton, M. G., Török, T., \& Karlický, M. 2010,

Sol. Phys., 266, 91

Kołomański, S., \& Karlický, M. 2007, A\&A, 475, 685

Kopp, R. A., \& Pneuman, G. W. 1976, Sol. Phys., 50, 85

Kuckein, C., Martínez Pillet, V., \& Centeno, R. 2012, A\&A, 539, A131

Labrosse, N., Heinzel, P., Vial, J.-C., et al. 2010, Space Sci. Rev., 151,243

Landi, E., Young, P. R., Dere, K. P., Del Zanna, G., \& Mason, H. E. 2013, ApJ, 763, 86

Lemen, J. R., Title, A. M., Akin, D. J., et al. 2012, Sol. Phys., 275,17

Lin, J., \& Forbes, T. G. 2000, J. Geophys. Res., 105, 2375

Liu, C., Lee, J., Karlický, M., et al. 2009, ApJ, 703, 757

Loureiro, N. F., Samtaney, R., Schekochihin, A. A., \& Uzdensky, D. A. 2012, Physics of Plasmas, 19, 042303

Lynch, B. J., Antiochos, S. K., DeVore, C. R., Luhmann, J. G., \& Zurbuchen, T. H. 2008, ApJ, 683, 1192

Magara, T., Mineshige, S., Yokoyama, T., \& Shibata, K. 1996, ApJ, 466, 1054

Masson, S., Aulanier, G., Pariat, E., \& Klein, K.-L. 2012, Sol. Phys., 276, 199

Masson, S., Pariat, E., Aulanier, G., \& Schrijver, C. J. 2009, ApJ, 700,559

McKenzie, D. E., \& Canfield, R. C. 2008, A\&A, 481, L65

Mészárosová, H., Dudík, J., Karlický, M., Madsen, F. R. H., \&

Sawant, H. S. 2013, Sol. Phys., 283, 473

Milligan, R. O., \& Dennis, B. R. 2009, ApJ, 699, 968 
Milligan, R. O., McAteer, R. T. J., Dennis, B. R., \& Young, C. A. 2010, ApJ, 713, 1292

Monstein, C. 2013, in EGU General Assembly Conference Abstracts, Vol. 15, EGU General Assembly Conference Abstracts, 2027

Moore, R. L., Schmieder, B., Hathaway, D. H., \& Tarbell, T. D. 1997, Sol. Phys., 176, 153

Moore, R. L., Sterling, A. C., Hudson, H. S., \& Lemen, J. R. 2001, ApJ, 552, 833

Neupert, W. M. 1968, ApJ, 153, L59

Ning, Z. J. 2011, in Astronomical Society of India Conference Series, Vol. 2, Astronomical Society of India Conference Series, 279

O’Dwyer, B., Del Zanna, G., Mason, H. E., Weber, M. A., \& Tripathi, D. 2010, A\&A, 521, A21

Ohyama, M., \& Shibata, K. 1998, ApJ, 499, 934

Pariat, E., \& Démoulin, P. 2012, A\&A, 541, A78

Parker, E. N. 1957, J. Geophys. Res., 62, 509

Patsourakos, S., Vourlidas, A., \& Stenborg, G. 2013, ApJ, 764, 125

Petkaki, P., Del Zanna, G., Mason, H. E., \& Bradshaw, S. J. 2012, A\&A, 547, A25

Petschek, H. E. 1964, NASA Special Publication, 50, 425

Poduval, B., DeForest, C. E., Schmelz, J. T., \& Pathak, S. 2013, ApJ, 765, 144

Priest, E., \& Forbes, T. 2000, Magnetic Reconnection

Priest, E. R., \& Démoulin, P. 1995, J. Geophys. Res., 1002, 23443

Priest, E. R., Hornig, G., \& Pontin, D. I. 2003, Journal of Geophysical Research (Space Physics), 108, 1285

Raftery, C. L., Gallagher, P. T., Milligan, R. O., \& Klimchuk, J. A. 2009, A\&A, 494, 1127

Reid, H. A. S., Vilmer, N., Aulanier, G., \& Pariat, E. 2012, A\&A, 547, A52

Savcheva, A., Pariat, E., van Ballegooijen, A., Aulanier, G., \& DeLuca, E. 2012, ApJ, 750, 15

Scherrer, P. H., Schou, J., Bush, R. I., et al. 2012, Sol. Phys., 275, 207

Schmelz, J. T., Jenkins, B. S., \& Pathak, S. 2013, ApJ, 770, 14

Schmelz, J. T., Reames, D. V., von Steiger, R., \& Basu, S. 2012, ApJ, 755, 33
Schmieder, B., Heinzel, P., van Driel-Gesztelyi, L., \& Lemen, J. R. 1996, Sol. Phys., 165, 303

Schrijver, C. J., Title, A. M., Yeates, A. R., \& DeRosa, M. L. 2013, ArXiv e-prints

Shen, Y., Liu, Y., \& Su, J. 2012, ApJ, 750, 12

Shibata, K., \& Tanuma, S. 2001, Earth, Planets, and Space, 53, 473

Sturrock, P. A. 1966, Nature, 211, 695

Sweet, P. A. 1958, in IAU Symposium, Vol. 6, Electromagnetic

Phenomena in Cosmical Physics, ed. B. Lehnert, 123

Testa, P., De Pontieu, B., Martínez-Sykora, J., et al. 2013, ApJ, 770, L1

Titov, V. S., Hornig, G., \& Démoulin, P. 2002, Journal of

Geophysical Research (Space Physics), 107, 1164

Török, T., Kliem, B., \& Titov, V. S. 2004, A\&A, 413, L27

Török, T., Panasenco, O., Titov, V. S., et al. 2011, ApJ, 739, L63

Uzdensky, D. A., Loureiro, N. F., \& Schekochihin, A. A. 2010

Physical Review Letters, 105, 235002

van Ballegooijen, A. A., \& Martens, P. C. H. 1989, ApJ, 343, 971

Warren, H. P., \& Warshall, A. D. 2001, ApJ, 560, L87

Wheatland, M. S. 2006, Sol. Phys., 236, 313

White, S. M., Benz, A. O., Christe, S., et al. 2011, Space Sci. Rev., 159, 225

Wilmot-Smith, A. L., Hornig, G., \& Pontin, D. I. 2009, ApJ, 704 1288

Wuelser, J.-P., Lemen, J. R., Tarbell, T. D., et al. 2004, in Society of Photo-Optical Instrumentation Engineers (SPIE) Conference Series, Vol. 5171, Society of Photo-Optical Instrumentation Engineers (SPIE) Conference Series, ed. S. Fineschi \& M. A. Gummin, 111-122

Yelles Chaouche, L., Kuckein, C., Martínez Pillet, V., \& Moreno-Insertis, F. 2012, ApJ, 748, 23

Young, P. R., Doschek, G. A., Warren, H. P., \& Hara, H. 2013, ApJ, 766, 127

Zhang, J., Cheng, X., \& Ding, M.-D. 2012, Nature

Communications, 3

Zuccarello, F., Romano, P., Farnik, F., et al. 2009, A\&A, 493, 629

Zweibel, E. G., \& Yamada, M. 2009, ARA\&A, 47, 291 